\title{
Vacuum Polarization in a Zero-Width Potential: Self-Adjoint Extension
}

\author{
Yuri V. Grats * and Pavel Spirin
}

Citation: Grats, Y.V.; Spirin, P. Vacuum Polarization in a Zero-Width Potential: Self-Adjoint Extension. Universe 2021, 7, 127. https:// doi.org/10.3390/universe7050127

Academic Editor: Galina L. Klimchitskaya

Received: 11 April 2021

Accepted: 27 April 2021

Published: 2 May 2021

Publisher's Note: MDPI stays neutral with regard to jurisdictional claims in published maps and institutional affiliations.

Copyright: (c) 2021 by the authors. Licensee MDPI, Basel, Switzerland. This article is an open access article distributed under the terms and conditions of the Creative Commons Attribution (CC BY) license (https:// creativecommons.org/licenses/by/ $4.0 /)$.
Physics Department, M.V.Lomonosov Moscow State University, Leninskie Gory 1/2, 119991 Moscow, Russia; salotop@list.ru

* Correspondence: grats@phys.msu.ru

\begin{abstract}
The effects of vacuum polarization associated with a massless scalar field near pointlike source with a zero-range potential in three spatial dimensions are analyzed. The "physical" approach consists in the usage of direct delta-potential as a model of pointlike interaction. We use the Perturbation theory in the Fourier space with dimensional regularization of the momentum integrals. In the weak-field approximation, we compute the effects of interest. The "mathematical" approach implies the self-adjoint extension technique. In the Quantum-Field-Theory framework we consider the massless scalar field in a 3-dimensional Euclidean space with an extracted point. With appropriate boundary conditions it is considered an adequate mathematical model for the description of a pointlike source. We compute the renormalized vacuum expectation value $\left\langle\phi^{2}(x)\right\rangle_{\text {ren }}$ of the field square and the renormalized vacuum averaged of the scalar-field's energy-momentum tensor

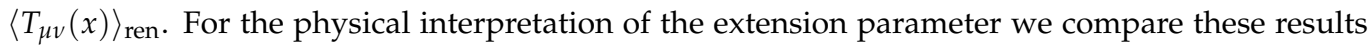
with those of perturbative computations. In addition, we present some general formulae for vacuum polarization effects at large distances in the presence of an abstract weak potential with finite-sized compact support.
\end{abstract}

Keywords: singular potential; self-adjoint extension; dimensional regularization; zero-width interaction; renormalization

\section{Introduction}

The standard problems of research within the quantum (field) theory, to which physicists return through decades, are the effects of the vacuum polarization on backgrounds with nontrivial local or global structure, boundaries and external fields. These problems in fact have a number of nontrivial features. The main of those is that the problems are determined by the solutions of partial differential equations on the manifold as a whole. So, the effects become essentially non-local. This non-locality leads to the non-trivial effects even when one considers the Euclidean space with a discrete number (finite or infinite) of subtracted points $y$. In this case the adequate boundary conditions enable to consider quantum systems given by Hamiltonian of the form

$$
H=-\Delta+\sum_{y} \lambda_{y} \delta^{d}(\boldsymbol{x}-\boldsymbol{y})
$$

where $\Delta$ is the self-adjoint Laplacian in $L^{2}\left(\mathbb{R}^{d}\right)$ with domain $H^{2,2}\left(\mathbb{R}^{d}\right), d=1,2,3$, is the dimensionality of the configuration space, $\delta^{d}(\boldsymbol{x}-\boldsymbol{y})$ is the Dirac $\delta$-function localized at $\boldsymbol{y}$, $\lambda_{y}$ is a corresponding coupling constant.

Models, which are heuristically given by Hamiltonian of the form (1), are known in the literature as "contact interaction models", "zero-range interaction models", "delta-like interaction models", "point interaction models" and "Fermi potential models". Models of this type have been widely discussed in physical and mathematical literature in connection with problems of solid-state physics (Kronig-Penney model [1]), atomic, and 
nuclear physics (description of short-range nuclear forces [2]). Encoding of the boundary conditions by a $\delta$-like potential made it possible to investigate the Casimir interaction of two parallel perfectly conducting plates [3-9], of two pointlike or linear sources with a zero-range potential [10-13]. Similar situations arise in consideration of field-theoretical effects near topological defects. Indeed, the space-time of an infinitely thin straight cosmic string has a conical structure with a $\delta$-like singularity at the top of a cone [14], and in suitable coordinates the equation for a massless scalar field with nonminimal coupling takes the form of Equation (1) but with a "curved" Laplacian $[15,16]$. Notice, already in consideration of FT-processes on the cosmic-string background, as well as within the analogous (2 + 1)-dimensional problems [17-20], it was crucial a question, in what sense one should regard the expression (1). In particular, by one way or another, it was proposed the smoothening of the singularity at conical apex, i.e., in fact to regard the $\lambda \delta$-potential as a distributional limit of an appropriate delta-like sequence [19-22]. Some conceptual problems of the $\lambda \delta$-potential introduction were discussed in $[23,24]$.

Similar singular configurations were previously analyzed in the recent literature. Among the notable are the effective action of pointlike interactions [25], the delta-potentials at finite temperature [26], Casimir interactions of manifolds with diverse codimensionalities [27], the Casimir effect with a boundary hyperplane [28], zeta regularization approach to various Casimir problems [10-12,29], and so forth.

It is known [30-32] that for $d \geqslant 4$ any self-adjoint extension (SAE) of the $H=-\Delta$, for which one uses the formal form $-\Delta+\lambda \delta$, is nothing but $-\Delta$. For $d=2,3$ the free operator $-\Delta$ is self-adjoint when acting on the finite functions. But for two and three dimensions $H$ remains to be self-adjoint even when the eigenfunctions blow up at $r=0$, preserving the local square-integrability. The self-adjointness of $H$ is guaranteed by the specification of boundary conditions. For these two dimensions there exits a one-parameter family of self-adjoint operators, labeled by a parameter of the self-adjoint extension $\alpha$, which is related with the renormalized value of the coupling constant $\lambda$. Namely, any operator of this family describes the $s$-wave zero-range scattering, while the corresponding scattering length is proportional to $-1 / \alpha[17,18,30,31]$. The basic difference of the twoand three-dimensional cases from the one-dimensional case is the fact that for $d=1$ the SAE-parameter $\alpha$ represents not the inverse scattering length but the coupling constant of the $\delta$-interaction. We will consider the case where the set of $\delta$-singularities consists of the single point-like source localized at the coordinate-system origin and restrict our consideration by the case $d=3$.

The paper is organized as follows-the Introduction is the first section. The perturbation theory we use is described in the Section 2-the approximated Green's function is constructed and preliminary results for the vacuum averages of interest are computed. Here we work within the approach proposed in $[15,16]$ and substitute the delta by a nonsingular function with finite-sized compact support; then we transit to the zero-width limit. Section 3 is devoted to the computation of the renormalized vacuum $\left\langle\phi^{2}(x)\right\rangle_{\text {ren }}$ and $\left\langle T_{\mu v}(x)\right\rangle_{\text {ren }}$ in the self-adjoint scheme. We use the direct mode summation to construct the Hadamard's function for the SAE of the Hamiltonian and points-splitting procedure for the computation of VEVs. We try to present our computational scheme and results in more detail. The renormalized EMT reproduces the result computed previously by Pizzocchero and Fermi [10] by zeta regularization technique. The comparison with the analogous results obtained in the perturbation theory method, is presented in the last Section 4, the Discussion. Here we summarize the results and point out some prospects.

We use the units $\hbar=c=1$ and metric with the signature $\eta_{\mu \nu}=\operatorname{diag}(1,-1,-1,-1)$. The notation $g_{\mu \nu}$ will denote the same Minkowski metric in arbitrary curvilinear coordinates.

\section{Perturbation Theory over Potential}

In the model we consider the quantized massless scalar field $\phi(t, x)$, living in the static $(3+1)$-dimensional bulk with the point-like spherically-symmetric potential located at the single point. With no loss of generality we set this point as origin of a coordinate system. 
We start with the Klein-Gordon-Fock equation for the massless scalar field in the presence of a static $\delta^{3}$-potential:

$$
\left[\partial^{2}+\lambda \delta^{3}(x)\right] \phi(t, x)=0, \quad \partial^{2} \equiv \eta^{\mu v} \partial_{\mu} \partial_{\nu}=\partial_{t}^{2}-\Delta,
$$

with $\lambda$ as constant real-valued coupling.

It is to be mentioned that $\delta$ should be regarded as no more than just a symbol until one decrypts the rule of its usage. We mention the following basic approaches to treat the $\delta$-symbol (it concerns derivatives of $\delta$ as well):

(i) $\delta$ may appear as a solution for some kernel of the subsequent integration (like in the Green's function); in this case the definition of actual usage of $\delta$ is formulated in terms of some continuous functional acting on the proper test function (a source);

(ii) $\delta$ may be considered as limiting state of locally integrable "common" functions (the delta-like sequences);

(iii) $\delta$ may be considered just as a rule, which extracts the locus of delta from consideration but sets some requirements on the admissible solutions and/or their derivatives (like in Quantum Mechanics).

In our problem of vacuum polarization we have no "source" to be integrated, so we pay attention to cases (ii) and (iii). Concerning case (ii), we shall consider some abstract arbitrary static potential $\mathcal{U}(\boldsymbol{x})$, with properties close to the pointlike delta. It is natural to set the requirement for $\mathcal{U}$ to be of finite size and have a compact support. Due to the arbitrariness we solve the problem iteratively by means of the Perturbation Theory.

The proper mathematical apparatus for case (iii), known in the literature on mathematical physics, implies the construction of a self-adjoint extension of the field operator $(-\Delta$ as $\partial^{2}$ restricted on the hyperplane $t=$ const in our case, which is a symmetric Hamiltonian). We postpone the computation by this type to Section 3 .

\subsection{Abstract Static Potential}

Let us start with perturbative calculations working with abstract potential $\mathcal{U}(x)$. It appears in the action as

$$
\mathscr{S}=\int d^{4} x\left[\partial_{\mu} \phi \partial^{\mu} \phi-\mathcal{U}(x) \phi^{2}\right] .
$$

If $\mathcal{U}=$ const $>0$ then it may be regarded as a mass square $\left(\mathcal{U}=m^{2}\right)$ and we return to the massive real scalar field. If the potential is a negative constant, we have a massive scalar field with tachyon. In what follows, the sign of value of the potential plays a crucial role. It also will be of emphatic significance in our consideration.

The action (3) has the following equation of motion:

$$
\left[\partial^{2}+\mathcal{U}(x)\right] \phi(t, x)=0 .
$$

The Feynman propagator for the scalar field satisfies the equation ${ }^{1}$

$$
\left[\partial^{2}+\mathcal{U}(x)\right] G^{F}\left(x, x^{\prime}\right)=-\delta\left(t-t^{\prime}\right) \delta^{3}\left(x-x^{\prime}\right) .
$$

Denoting the field operator in the coordinate representation as $\mathcal{L}(x) \equiv \partial^{2}+\mathcal{U}(x)$ and representing the corresponding equation in the Schwinger (operator) form

$$
\mathfrak{L} \mathfrak{G}=-1,
$$

where

$$
\left\langle x|\mathfrak{L}| x^{\prime}\right\rangle \equiv \mathcal{L}(x) \delta\left(x-x^{\prime}\right), \quad G^{F}\left(x, x^{\prime}\right) \equiv\left\langle x|\mathfrak{G}| x^{\prime}\right\rangle,
$$

1 In what follows we define the Feynman propagator as $G^{F}\left(x, x^{\prime}\right)=-i\left\langle T\left[\phi(x) \phi\left(x^{\prime}\right)\right]\right\rangle_{\text {vac }}$. 
it admits the operator solution $\mathfrak{G}=-\mathfrak{L}^{-1}$. If operator $\mathfrak{L}$ allows to be expressed as $\mathfrak{L}=\mathfrak{L}_{0}+\mathfrak{U}$, where $\mathfrak{U}$ is considered as a small perturbation (in some sense) of $\mathfrak{L}_{0}$, one can represent the solution to Equation (4) in the form

$$
\mathfrak{G}=\left[-\mathfrak{L}_{0}\left(1-\mathfrak{G}_{0} \mathfrak{U}\right)\right]^{-1}=\mathfrak{G}_{0}+\mathfrak{G}_{0} \mathfrak{U} \mathfrak{G}_{0}+\mathfrak{G}_{0} \mathfrak{U} \mathfrak{G}_{0} \mathfrak{U} \mathfrak{G}_{0}+\ldots .
$$

with $\mathfrak{G}_{0}=-\mathfrak{L}_{0}{ }^{-1}$.

In the case under consideration $\mathfrak{L}_{0}=\partial^{2}$ is determined by the zeroth order in the small "quantity" $U \ll 1$ (somehow directly related with the small operator $\mathcal{U}$ ). In the problem-at-hand the function $G_{0}^{F}\left(x, x^{\prime}\right)=\left\langle x\left|\mathfrak{G}_{0}\right| x^{\prime}\right\rangle=-\left\langle x\left|\partial^{-2}\right| x^{\prime}\right\rangle$ in Fourier basis takes the form ${ }^{2}$ :

$$
G_{0}^{F}\left(x-x^{\prime}\right)=\int \frac{d^{4} p}{(2 \pi)^{4}} \frac{\mathrm{e}^{-i p\left(x-x^{\prime}\right)}}{p^{2}+i \varepsilon}
$$

and for the first-order correction to the Green's function from (5) we get the following expression:

$$
G_{1}^{F}\left(x, x^{\prime}\right)=\left\langle x\left|\mathfrak{G}_{0} \mathfrak{U} \mathfrak{G}_{0}\right| x^{\prime}\right\rangle=\int \frac{d^{4} q}{(2 \pi)^{4}} \mathrm{e}^{-i q x} \mathcal{U}(q) \int \frac{d^{4} p}{(2 \pi)^{4}} \frac{\mathrm{e}^{-i p\left(x-x^{\prime}\right)}}{\left(p^{2}+i \varepsilon\right)\left[(p+q)^{2}+i \varepsilon\right]},
$$

where $q^{\mu}=\left(q^{0}, \boldsymbol{q}\right), p^{\mu}=\left(p^{0}, \boldsymbol{p}\right) ; \boldsymbol{q}$ and $\boldsymbol{p}$ are 3-dimensional Euclidean vectors.

\subsection{First-Order VEVs for Static Potential}

If the perturbation operator depends on the spatial coordinates only (that is, the case in our problem), its Fourier-transform reads

$$
\mathcal{U}(q)=2 \pi \delta\left(q^{0}\right) \mathcal{U}(\boldsymbol{q}),
$$

and from (7) we get

$$
G^{F}\left(x, x^{\prime}\right)=G_{0}^{F}+\int \frac{d^{3} q}{(2 \pi)^{3}} \mathrm{e}^{i \boldsymbol{q} x} \mathcal{U}(\boldsymbol{q}) \int \frac{d^{4} p}{(2 \pi)^{4}} \frac{\mathrm{e}^{-i p\left(x-x^{\prime}\right)}}{\left(p^{2}+i \varepsilon\right)\left[p_{0}^{2}-(\boldsymbol{p}+\boldsymbol{q})^{2}+i \varepsilon\right]}+\mathcal{O}\left(\mathcal{U}^{2}\right) .
$$

All the quantities we are interested in, are expressed via the Feynman propagator $G^{F}\left(x, x^{\prime}\right)$ and its derivatives, evaluated in coincident points [33]. The contribution of $G_{0}^{F}\left(x, x^{\prime}\right)(6)$ contains the single propagator and vanishes in regularization (see e.g., the Appendix of [15]); we refer these terms as to "tadpole"-contributions.

In terms of $G^{F}\left(x, x^{\prime}\right)$, the vacuum field-square average reads

$$
\left\langle\phi^{2}(x)\right\rangle_{\mathrm{reg}}=i \lim _{x^{\prime} \rightarrow x} G^{F}\left(x, x^{\prime}\right)_{\mathrm{reg}} \equiv i \lim _{x^{\prime} \rightarrow x} G_{1}^{F}\left(x, x^{\prime}\right) .
$$

Thus we obtain, that with our accuracy

$$
\left\langle\phi^{2}(x)\right\rangle_{\text {reg }}=i \int \frac{d^{3} q}{(2 \pi)^{3}} \mathrm{e}^{i \boldsymbol{q} x} \mathcal{U}(\boldsymbol{q}) \int \frac{d^{4} p}{(2 \pi)^{4}} \frac{1}{\left(p^{2}+i \varepsilon\right)\left[(p+\tilde{q})^{2}+i \varepsilon\right]},
$$

where $\tilde{q}^{\mu}=(0, \boldsymbol{q})$ was introduced to keep the 4-dimensional covariant form. The corresponding expressions diverge, and for their evaluation we make use of the dimensional-

2 Hereafter the 4- and 3-dimensional direct Fourier-transforms are defined as

$$
\varphi(q)=\int d^{4} x \varphi(x) \mathrm{e}^{i q x}, \quad \varphi(\boldsymbol{q})=\int d^{3} x \varphi(x) \mathrm{e}^{-i \boldsymbol{q} x} .
$$


regularization method. The internal integral with two propagators has a standard form in the dimensional-regularization scheme (see e.g., [34]), and the regularized value is given by

$$
J(q) \equiv \int \frac{d^{D} p}{(2 \pi)^{D}} \frac{1}{\left(p^{2}+i \varepsilon\right)\left[(p+q)^{2}+i \varepsilon\right]}=i \frac{\Gamma^{2}\left(\frac{D}{2}\right) \Gamma\left(-\frac{D-4}{2}\right)}{(4 \pi)^{D / 2} \Gamma(D-2)}\left|q^{2}\right|^{D / 2-2},
$$

which for $D=4-2 \delta$ and $q=\tilde{q}$ yields

$$
J(|\boldsymbol{q}|)=i \frac{\Gamma(\delta)}{(4 \pi)^{2}|\boldsymbol{q}|^{2 \delta}}[1+\mathcal{O}(\delta)] .
$$

Plugging it into (9) one obtains

$$
\left\langle\phi^{2}(x)\right\rangle_{\mathrm{reg}}=-\frac{\Gamma(\delta)}{(4 \pi)^{2}} \int \frac{d^{3} q}{(2 \pi)^{3}} \mathrm{e}^{i \boldsymbol{q} x} \frac{\mathcal{U}(\boldsymbol{q})}{|\boldsymbol{q}|^{2 \delta}},
$$

which has a form of an inverse 3-dimensional Fourier-transform, but with infinite prefactor $\Gamma(\delta)$ (as $\delta \rightarrow+0$ ). To fix it, represent $\mathcal{U}(\boldsymbol{q})$ as a 3-dimensional Fourier-transform with respect to new variable $x^{\prime}$.

Integrating over $q$ with help of the table Fourier-transform [35]

$$
\int d^{n} q \frac{e^{i \boldsymbol{q} y}}{|\boldsymbol{q}|^{\lambda}}=\frac{2^{n-\lambda} \pi^{n / 2}}{|\boldsymbol{y}|^{n-\lambda}} \frac{\Gamma\left(\frac{n-\lambda}{2}\right)}{\Gamma\left(\frac{\lambda}{2}\right)}
$$

one has

$$
\int d^{3} q \frac{e^{i \boldsymbol{q} y}}{|\boldsymbol{q}|^{2 \delta}}=\frac{4 \pi^{2}}{\Gamma(\delta)} \frac{1}{|\boldsymbol{y}|^{3}}[1+\mathcal{O}(\delta)],
$$

so we check that undesirable $\Gamma(\delta)$ have disappeared. Therefore after the regularization removal $(\delta=0)$ one arrives at

$$
\left\langle\phi^{2}(x)\right\rangle_{\text {ren }}=-\frac{1}{32 \pi^{3}} \int d^{3} x^{\prime} \frac{\mathcal{U}\left(x^{\prime}\right)}{\left|x-x^{\prime}\right|^{3}} .
$$

The integral is obviously convergent if the potential has a compact support and we consider the observation points outside it. In this case Equation (13) has a form, reminiscent to the zeroth-order term of the multipolar expansion of electrostatic potential. Nevertheless, the integral diverges for the observation points inside the compact potential. Therefore if $R$ is a size of the potential's support, we get an apparent requirement

$$
r>R \text {. }
$$

However, this condition may become even more stronger if take into account the possible convergence of the whole series: indeed, it may appear some another lengthy constants from the concrete problem-at-hand.

Renormalized EMT. Following the same ideology, we compute the VEV for the components of the energy-momentum tensor of the massless real-valued scalar field. In terms of the Green's function, the regularized vacuum expectation value of the energymomentum tensor in a flat space is given by

$$
\left\langle T_{\mu}^{v}\right\rangle_{\text {ren }}=i \lim _{x^{\prime} \rightarrow x} D_{\mu}^{v} G^{F}\left(x, x^{\prime}\right)_{\text {reg }} \equiv i \lim _{x^{\prime} \rightarrow x} D_{\mu}^{v} G_{1}^{F}\left(x, x^{\prime}\right),
$$


where $D_{\mu}^{\nu}$ stands for the appropriate differential operator $[33,36]\left(\nabla_{\mu}\right.$ and $\nabla_{\mu}^{\prime}$ denote the covariant derivative over $x^{\mu}$ and $x^{\prime \mu}$, respectively, while $\partial_{\mu}$ and $\partial_{\mu}^{\prime}$ stand for the corresponding ordinary derivatives) with no curvature:

$$
D_{\mu}^{v}=(1-2 \xi) \partial^{v} \partial_{\mu}^{\prime}-\left(\frac{1}{2}-2 \xi\right) \delta_{\mu}^{v} \partial^{\lambda} \partial_{\lambda}^{\prime}-\xi\left(\nabla^{v} \partial_{\mu}+\nabla^{\prime v} \partial_{\mu}^{\prime}\right)+\left(2 \xi \nabla^{\lambda} \partial_{\lambda}-\frac{\mathcal{U}}{2}\right) \delta_{\mu}^{v} .
$$

Notice, despite the spacetime is flat, the coordinate system may be non-Cartesian, and the Christoffel symbols may well survive. Therefore we keep the covariant derivatives as well.

Therefore the first-order (with respect to $\mathcal{U}$ ) contribution to the EMT consists of two parts: the first, which already sits in the operator $D_{\mu}^{v}(14)$, and the second, which appears as the first correction to the Feynman propagator times the non- $\mathcal{U}$ terms in Equation (14). For self-consistency, the first contribution should be computed with the help of the zeroth-order Feynman propagator $G_{0}^{F}\left(x, x^{\prime}\right)$, whose coincidence-point limit corresponds to the tadpole diagram and has to be neglected. Thus we are left with the second-type contribution.

Acting by (14) on (7) and taking the coincidence-point limit, one obtains for the vacuum energy density:

$$
\begin{aligned}
\left\langle T_{00}\right\rangle= & i \int \frac{d^{4} q}{(2 \pi)^{4}} \mathrm{e}^{-i q x} \mathcal{U}(q) \times \\
& \times \int \frac{d^{4} p}{(2 \pi)^{4}} \frac{p_{0}^{2}+p_{0} q_{0}+\xi q_{0}^{2}+(2 \xi-1 / 2)\left(p^{2}+p \cdot q\right)-2 \xi(p+q)^{2}}{\left(p^{2}+i \varepsilon\right)\left[(p+q)^{2}+i \varepsilon\right]} .
\end{aligned}
$$

Making use of the fact that the potential is static (Equation (8)), all terms containing $q_{0}$, disappear from (15). As before, the internal integral is evaluated within the dimensionalregularization technique, where the $p^{2}$ - and $(p+q)^{2}$-terms in numerator are considered as tadpole-like ones and thus should be neglected. By this reason, we may completely ignore the last term in (14). Therefore the non-vanishing contribution reads

$$
\left\langle T_{00}\right\rangle=i \int \frac{d^{3} q}{(2 \pi)^{3}} \mathrm{e}^{i \boldsymbol{q} x} \mathcal{U}(\boldsymbol{q}) \int \frac{d^{4} p}{(2 \pi)^{4}} \frac{p_{0}^{2}+(2 \tilde{\zeta}-1 / 2) p \cdot \tilde{q}}{\left(p^{2}+i \varepsilon\right)\left[(p+\tilde{q})^{2}+i \varepsilon\right]} .
$$

For the internal integration we encounter the vectorial and tensorial two-propagator integrals, also familiar for the dimensional regularization (see, e.g., the textbooks $[34,37]$ on QFT or Appendix of [15]); their regularized values are

$$
\begin{aligned}
J_{\mu} & \equiv \int \frac{d^{D} p}{(2 \pi)^{D}} \frac{p_{\mu}}{\left(p^{2}+i \varepsilon\right)\left[(p+q)^{2}+i \varepsilon\right]}=-\frac{J}{2} q_{\mu}, \\
J_{\mu \nu} & \equiv \int \frac{d^{D} p}{(2 \pi)^{D}} \frac{p_{\mu} p_{v}}{\left(p^{2}+i \varepsilon\right)\left[(p+q)^{2}+i \varepsilon\right]}=-\frac{J}{4(D-1)}\left(q^{2} g_{\mu \nu}-D q_{\mu} q_{\nu}\right),
\end{aligned}
$$

(where $J$ is given by (11) in our case), to obtain

$$
\left\langle T_{00}\right\rangle_{\text {ren }}=-\frac{\Gamma(\delta)}{(4 \pi)^{2}}\left(\xi-\frac{1}{6}\right) \int \frac{d^{3} q}{(2 \pi)^{3}} \mathrm{e}^{i \boldsymbol{q} x} \mathcal{U}(\boldsymbol{q})|\boldsymbol{q}|^{2-2 \delta} .
$$

Operating along the same lines as for $\left\langle\phi^{2}(x)\right\rangle_{\text {ren }}$, we arrive at the following coordinaterepresentation formula

$$
\left\langle T_{00}(x)\right\rangle_{\text {ren }}=\frac{3}{16 \pi^{3}}\left(\xi-\frac{1}{6}\right) \int d^{3} x^{\prime} \frac{\mathcal{U}\left(x^{\prime}\right)}{\left|x-x^{\prime}\right|^{5}} .
$$


For the spatial components of the renormalized EMT with lowercase indices we have (in cartesian coordinates) the following non-vanishing expression:

$$
\begin{aligned}
\left\langle T_{i j}\right\rangle= & -i \int \frac{d^{3} q}{(2 \pi)^{3}} \mathrm{e}^{i \boldsymbol{q} x} \mathcal{U}(\boldsymbol{q}) \times \\
& \times \int \frac{d^{4} p}{(2 \pi)^{4}} \frac{(\xi-1) \tilde{q}_{i} p_{j}-p_{i} p_{j}+(2 \xi-1 / 2) p \cdot \tilde{q} \delta_{i j}-\xi\left(p_{i} \tilde{q}_{j}+\tilde{q}_{i} \tilde{q}_{j}\right)}{\left(p^{2}+i \varepsilon\right)\left[(p+\tilde{q})^{2}+i \varepsilon\right]} .
\end{aligned}
$$

After the computation of the internal integral one obtains:

$$
\left\langle T_{i j}\right\rangle=-i\left(\xi-\frac{1}{6}\right) \int \frac{d^{3} q}{(2 \pi)^{3}} \mathrm{e}^{i \boldsymbol{q} x} \mathcal{U}(\boldsymbol{q}) J(|\boldsymbol{q}|)\left(\boldsymbol{q}^{2} \delta_{i j}-q_{i} q_{j}\right) .
$$

The first term in the parentheses, after the subsequent integration, equals $\left\langle T_{00}\right\rangle_{\text {ren }} \delta_{i j}$. For the second one we differentiate (12): ${ }^{3}$

$$
\begin{aligned}
\int d^{n} q \frac{e^{i \boldsymbol{q} y}}{|\boldsymbol{q}|^{\lambda}} q_{i} q_{j} & =-\frac{\partial^{2}}{\partial y^{i} \partial y^{j}} \frac{2^{n-\lambda} \pi^{n / 2}}{|\boldsymbol{y}|^{n-\lambda}} \frac{\Gamma\left(\frac{n-\lambda}{2}\right)}{\Gamma\left(\frac{\lambda}{2}\right)}= \\
& =\frac{2^{n-\lambda+1} \pi^{n / 2}}{|\boldsymbol{y}|^{n-\lambda+2}} \frac{\Gamma\left(\frac{n-\lambda+2}{2}\right)}{\Gamma\left(\frac{\lambda}{2}\right)}\left(\delta_{i j}-\frac{(n-\lambda+2) y_{i} y_{j}}{y^{2}}\right) .
\end{aligned}
$$

Combining all terms, the renormalized spatial sector of the EMT is given by

$$
\left\langle T_{i j}(x)\right\rangle_{\text {ren }}=\frac{3}{32 \pi^{3}}\left(\xi-\frac{1}{6}\right) \int d^{3} x^{\prime} \frac{\mathcal{U}\left(x^{\prime}\right)}{\left|x-x^{\prime}\right|^{5}}\left(5 \frac{x_{i} x_{j}-2 x_{i} x_{j}^{\prime}+x_{i}^{\prime} x_{j}^{\prime}}{\left|x-x^{\prime}\right|^{2}}-3 \delta_{i j}\right) .
$$

The mixed components in cartesian coordinates are determined by the following effective expression:

$$
\left\langle T_{0 i}\right\rangle=i \int \frac{d^{3} q}{(2 \pi)^{3}} \mathrm{e}^{i \boldsymbol{q} x} \mathcal{U}(\boldsymbol{q}) \int \frac{d^{4} p}{(2 \pi)^{4}} \frac{(\xi-1) p_{0} \tilde{q}_{i}-p_{0} p_{i}}{\left(p^{2}+i \varepsilon\right)\left[(p+\tilde{q})^{2}+i \varepsilon\right]},
$$

which, upon the $p$-integration, reduces to superposition of $\tilde{q}_{0} \tilde{q}_{i}$ - and $\eta_{0 i}$-monomials. Both them vanish:

$$
\left\langle T_{0 i}\right\rangle_{\text {ren }}=0 .
$$

Hence we conclude that the total EMT, to the first order of PT, is proportional to the factor $(\xi-1 / 6)$. It implies that for the conformal coupling the EMT does not vanish completely, but just scales at large distances not as $r^{-5}$, but at least as $r^{-6}$ or even weaker. Thus for the conformal case one requires the computations in the next order of PT, what is beyond our goal here. We advertise the confirmation of these notes in the next Section, being applied to the particular case of pointlike interaction.

The trace can be computed from cartesian coordinates: the spatial-sector trace is given by

$$
\begin{aligned}
\delta^{i j}\left\langle T_{i j}(x)\right\rangle_{\text {ren }} & =\frac{3}{8 \pi^{3}}\left(\xi-\frac{1}{6}\right) \int d^{3} x^{\prime} \frac{\mathcal{U}\left(x^{\prime}\right)}{\left|x-x^{\prime}\right|^{5}} \\
& =-2\left\langle T_{00}(x)\right\rangle_{\text {ren }} .
\end{aligned}
$$

3 Here $\boldsymbol{q}$ is $n$-dimensional Euclidean vector and $q_{i}$ stands for its $i$-th component. In Equation (18) the monomial $q_{i} q_{j}$ (or, equivalently, $\tilde{q}_{i} \tilde{q}_{j}$ ) is constructed from the components of the Minkowski vector $q^{\mu}$, where the sign changes in dependence of the index kind. But since $q$ enters quadratically, the lowercase Minkowski tensor $q_{i} q_{j}$ is identical to the Euclidean $q_{i} q_{j}$, where $\boldsymbol{q}$ is the spatial sector of $q^{\mu}$ with uppercase index. This equality does not give rise to any confusion. 
In what follows, the total trace equals

$$
\begin{aligned}
\operatorname{Sp}\langle T(x)\rangle_{\text {ren }} & =-\frac{9}{16 \pi^{3}}\left(\xi-\frac{1}{6}\right) \int d^{3} x^{\prime} \frac{\mathcal{U}\left(x^{\prime}\right)}{\left|x-x^{\prime}\right|^{5}} \\
& =3\left\langle T_{00}(x)\right\rangle_{\text {ren }} .
\end{aligned}
$$

Therefore for any static potential with compact support the trace is thrice the temporal component (to the first order with respect to the small potential).

The conservation law of the constructed renormalized energy-momentum tensor reduces to the spatial sector, given by (18), since $\left\langle T_{00}\right\rangle_{\text {ren }}$ does not depend upon time and all mixed components vanish $\left(\left\langle T_{0 i}\right\rangle_{\text {ren }}=0\right)$. Computing the spatial divergence

$$
\partial^{j}\left\langle T_{i j}(x)\right\rangle_{\text {ren }}=-\frac{3}{32 \pi^{3}}\left(\xi-\frac{1}{6}\right) \int d^{3} x^{\prime} \mathcal{U}\left(x^{\prime}\right) \frac{\partial}{\partial x_{j}}\left(5 \frac{\left(x_{i}-x_{i}^{\prime}\right)\left(x_{j}-x_{j}^{\prime}\right)}{\left|x-x^{\prime}\right|^{7}}-3 \frac{\delta_{i j}}{\left|x-x^{\prime}\right|^{5}}\right),
$$

one easily verifies that the expression vanishes already in the integrand, thus

$$
\partial^{v}\left\langle T_{\mu v}(\boldsymbol{x})\right\rangle_{\text {ren }}=0 .
$$

\subsection{Weak-Field Approximation for Zero-Range Potential}

The simplest case of the static spherically-symmetric potential is where the support consists of the single point; with no loss of generality we may choose it as origin of the coordinate system:

$$
\operatorname{supp} \mathcal{U}(r)=\{\boldsymbol{0}\} .
$$

According to the main theorem of the Theory of generalized functions [35], the tempered distribution concentrated on the single point, represents the finite sum of the derivatives of delta-function with locus at this point:

$$
g(x)=\sum_{k=0}^{N} c_{\alpha_{k} \beta_{k} \gamma_{k}} \frac{\partial^{k}}{\partial x^{\alpha_{k}} \partial y^{\beta_{k}} \partial z \gamma_{k}} \delta^{3}(x), \quad \quad \alpha_{k}+\beta_{k}+\gamma_{k}=k
$$

The corresponding restriction on the spherically-symmetric generalized functions reduces the condition to

$$
g(r)=\sum_{k=0}^{N} c_{k}(r) \delta_{+}^{(k)}(r), \quad 0 \leqslant N<\infty,
$$

where the "half-axis delta-function" $\delta_{+}(r)$ is defined as

$$
\int_{0}^{\infty} \delta_{+}(r) f(r) d r=f(0) .
$$

Now we restrict ourselves by the simplest case of this class, with $N=0$. The potential is given by

$$
\mathcal{U}(\boldsymbol{x})=\lambda \delta^{3}(\boldsymbol{x})=\frac{\lambda \delta_{+}(r)}{4 \pi r^{2}}
$$

in cartesian and spherical coordinates, respectively. Thereby the coefficient $\lambda$ plays a role of the potential's strength.

Apparently, for $\lambda>0$ we deal with repulsion, while the $\lambda<0$ is the case of attraction. If we consider the Schrödinger equation for the quantum non-relativistic "unit" mass $m=1 / 2$ in a spherical finite-size potential well with width $a$ (radius) and depth $3|\lambda| /\left(4 \pi a^{3}\right)$ inside the well, then in the limit $a \rightarrow+0$ the well becomes infinitely narrow and infinitely deep. For $\lambda>0$ we have the limiting case of the potential barrier. 
From dimensional-analysis consideration (in $\hbar=1$ units), one has

$$
\left[\lambda \delta^{3}(x)\right]=[\lambda] L^{-3}=[\square]=\left[L^{-2}\right]
$$

and thus $\lambda$ has a dimensionality of length. The usage of the perturbation theory implies that $|\mathcal{U}|=|\lambda| \delta^{3}(\boldsymbol{x})$ is "sufficiently small". Translating it to $\lambda$ as to the only parameter, we conclude that $\lambda$ is to be sufficiently small with respect to some another length parameter. It is not hard to deduce that the only such a parameter in the problem-at-hand, is the radial distance $r$, since the problem has radial symmetry and no other lengthy parameters are involved. Therefore the self-consistence of the PT usage is (naively)

$$
U=\frac{r}{|\lambda|}, \quad r \gg|\lambda| .
$$

In the sequel we will test this requirement, deduced heuristically.

Now we see that we started from the case (ii) of interpretation of $\delta$, but in the process of computation we get actually the potential as a source test function to be integrated with some kernel (Equations (13), (17) and (18), etc.). What corresponds to the paradigm (i) discussed above in the beginning of the Section.

Due to the delta-function, the application of the generic formulae, obtained above, is straightforward. For the renormalized field-square, we plug (21) into (13) to obtain:

$$
\left\langle\phi^{2}(r)\right\rangle_{\text {ren }}=-\frac{\lambda}{32 \pi^{3} r^{3}} \text {. }
$$

The renormalized vacuum energy density is obtained from the generic static Formula (17); computing, we get:

$$
\left\langle T_{00}(r)\right\rangle_{\text {ren }}=\frac{3 \lambda}{16 \pi^{3} r^{5}}\left(\xi-\frac{1}{6}\right)
$$

Substituting (21) into (18), the spatial components of the renormalized EMT are given by

$$
\left\langle T_{i j}(x)\right\rangle_{\text {ren }}=\frac{3 \lambda}{32 \pi^{3} r^{7}}\left(\xi-\frac{1}{6}\right)\left(5 x_{i} x_{j}-3 r^{2} \delta_{i j}\right)
$$

Transforming the tensor to spherical coordinates, the renormalized EMT with mixed indices reads:

$$
\left\langle T_{\mu}^{v}(t, r, \theta, \varphi)\right\rangle_{\text {ren }}=\frac{\lambda(6 \xi-1)}{32 \pi^{3} r^{5}} \operatorname{diag}\left(1,-1, \frac{3}{2}, \frac{3}{2}\right) .
$$

Therefore, to the first order of the Perturbation Theory, the renormalized EMT of the conformal field vanishes. Computing the trace directly from here, or making use of Equation (20), the total trace equals

$$
\left\langle T_{\mu}^{\mu}\right\rangle_{\text {ren }}=\frac{3 \lambda(6 \xi-1)}{32 \pi^{3} r^{5}}
$$

Now return to the problem of convergence and validity of the technique. We notice:

1. The effect vanishes in the limit $r \rightarrow \infty$, hence the weak-field approximation works at least at very high (with respect to some lengthy constant) distances;

2. The only such a constant for the problem-at-hand is $\lambda$, deduced above;

3. From dimensional consideration, the 2nd-order correction to $\left\langle\phi^{2}(r)\right\rangle_{\text {ren }}$ is expected to be of order $\mathcal{O}\left(\lambda^{2} / r^{4}\right)$. In other words, the parameter of formal series is $\lambda / r$.

Combining all them, we therefore expect the convergence at $\lambda / r \ll 1$. 
Summary: Within the Perturbation Theory, we derived some general formulae for the vacuum polarization of the massless scalar field in the presence of a static potential. We got, that for any static potential, the ratio of the trace of the renormalized energy-momentum tensor ${ }^{4}$ and its temporal component equals 3 , independent of the potential distribution. The generic expressions were applied to the case of zero-width potential. Nevertheless, as it is well-known, the perturbative solution may produce the series converging everywhere, or to give the asymptotic series, or the series with finite (or zero) convergence radius. Therefore the methods which may yield the non-perturbative solution, are of particular importance.

\section{Self-Adjoint Extension}

More satisfactory, especially within a mathematically-correct framework, the consideration of point-like interaction should be considered as a self-adjoint extension of a non-interacting Hamiltonian, defined on a space with the extracted point of the potential's locus. The parameter of such extension (if it is unique) could be related with the strength of this interaction.

In what follows, now we consider the non-negative operator $H=-\Delta$ defined on $\mathbb{R}^{3} \backslash\{\mathbf{0}\}$. The Klein-Gordon-Fock equation with the extracted origin reads:

$$
\square \phi(t, x)=0 \quad|x|>0 .
$$

As it was seen in the previous Section, the vacuum polarization of the massless field in this potential represents the effect, which is (i) static, (ii) spherically-symmetric, and (iii) rapidly decays with growth of a radial distance. Therefore the quantum-field effects are expressed in terms of the modes with no temporal-coordinate influence and free-wave behavior at infinity. In other words, we are in the situation where the difference between the QFT and Non-relativistic Quantum mechanics looks insufficient. Thus it is natural to consider the problem in the framework of Quantum Mechanics. To proceed, we factorize the time-dependence of a scalar field in a standard way by the following ansatz:

$$
\phi_{\omega}^{( \pm)}(t, x)=e^{\mp i \omega t} u_{\omega}(x)
$$

which distinguishes the positive- and negative-frequency solutions. Plugging it into Equation (27) one obtains

$$
H u_{\omega}(\boldsymbol{x})=\omega^{2} u_{\omega}(\boldsymbol{x}) .
$$

Consequently, we get the equation on the eigenvalues and eigenfunctions of the appropriate non-relativistic Hamiltonian. Equation (28) has a form of non-relativistic Schrödinger equation for a quantum-mechanical particle $(m=1 / 2)$ under the influence of a contact potential created by a point source located at the origin. Parameter $\lambda$ plays a role of the strength of interaction, while $\omega^{2}$ has physical meaning as the energy of a wave.

Since we deal with the spherically-symmetric potential, we introduce spherical coordinates, where the Laplacian has well-known decomposition on the spherical harmonics. Thus one obtains the resolution of the operator's definition space:

$$
L^{2}\left(\mathbb{R}^{3}\right)=\bigoplus_{l=0}^{\infty} L^{2}\left((0, \infty) ; r^{2} d r\right) \bigotimes\left[Y_{l,-l}, \ldots, Y_{l, l}\right],
$$

where $L^{2}\left((0, \infty) ; r^{2} d r\right)$ (standard denotation) means the set of functions, which are locally quadratically-integrable on $\mathbb{R}_{+}$with the measure $r^{2}$. Dots $[\ldots]$ denote a linear span of the vectors in brackets.

\footnotetext{
4 Excepting the conformal field.
} 
With respect to this decomposition, the closure of $H$ in $L^{2}\left(\mathbb{R}^{3}\right), \dot{H}$, equals the direct sum ${ }^{5}$

$$
\dot{H}=\bigoplus_{l=0}^{\infty} \dot{H}_{l} \bigotimes \mathbf{1},
$$

where the "partial Hamiltonians" are defined as

$$
\dot{H}_{l}=-\frac{d^{2}}{d r^{2}}-\frac{2}{r} \frac{d}{d r}+\frac{l(l+1)}{r^{2}}, \quad l=0,1,2, \ldots
$$

By the well-known results $[30,31]$, for $l \geqslant 1$ each operator $\dot{H}_{l}$ is self-adjoint itself, whereas the operator $\dot{H}_{0}$ admits a family of self-adjoint extensions $H_{0 \alpha}$ labeled by single real-valued parameter $\alpha$ :

$$
H_{0 \alpha}=-\frac{d^{2}}{d r^{2}}-\frac{2}{r} \frac{d}{d r}
$$

with the following domain of definition:

$$
\mathcal{D}\left(H_{0 \alpha}\right)=\left\{u_{\alpha} \in L^{2}\left((0, \infty) ; r^{2} d r\right) ; 4 \pi \alpha \lim _{r \rightarrow 0^{+}} r u_{\alpha}(r)=\lim _{r \rightarrow 0^{+}}\left(u_{\alpha}+r u_{\alpha}^{\prime}\right)\right\} .
$$

Thus we deal with the same operator $H_{0}$, but defined on the extended space of functions. Each extension is labeled by the single real-valued extension parameter $\alpha$, which, eventually, will restrict the possible eigenfunctions (labeled by pairwise index $u_{\omega \alpha}$ ) of a Hamiltonian.

Now we can go back and combine the self-adjoint $H_{0 \alpha}$ with other $\dot{H}_{l}(l \geqslant 1)$ and obtain the analogue of resolution (29) for the total extended Hamiltonian as a completely self-adjoint operator:

$$
H_{\alpha}=\left(H_{0 \alpha} \oplus \bigoplus_{l=1}^{\infty} \dot{H}_{l}\right) \bigotimes 1
$$

In these equations the parameter of self-adjoint extension $\alpha$ takes the values $-\infty<\alpha \leqslant \infty$, where the case " $\alpha=\infty$ " corresponds to the free Hamiltonian. The boundary condition allows for the wave eigenfunctions with $l=0$ to blow up at the origin, preserving for them to be still squared-integrable locally. The set of eigenfunctions, corresponding to the higher harmonics $\left(Y_{l m}\right.$, for $\left.l \geqslant 1\right)$, is the same as for free the Laplacian.

The rigorous relation of the abstract extension parameter $\alpha$ with the original strength parameter $\lambda$ of the delta-potential is to be detected below.

Therefore the change due to SAE may happen in the eigenfunctions of $H_{0}$, i.e., for $l=0$; that quantum state is traditionally marked by letter $s$. The corresponding modified solution is then called the "s-wave".

\subsection{Eigenfunctions and the Green's Function}

Given that the $s$-wave corresponds to $l=0$, whose angular part is trivial $Y_{00}=1$ and spherically-symmetric, now proceed to the solutions to the radial part of the partial extended Hamiltonian with $l=0$ :

$$
H_{0 \alpha} u_{\omega \alpha}(r)=\omega^{2} u_{\omega \alpha}(r) .
$$

Substitution of (30) into Equation (32) yields:

$$
u_{\omega \alpha}^{\prime \prime}(r)+\frac{2}{r} u_{\omega \alpha}^{\prime}+\omega^{2} u_{\omega \alpha}=0 .
$$

\footnotetext{
5 For the mathematically satisfactory consideration of the problem and more details see [31,32] and literature therein.
} 
Introducing $\chi_{\omega \alpha}(r)$ by $u_{\omega \alpha}=\chi_{\omega \alpha}(r) / r$, one obtains $\chi_{\omega \alpha}^{\prime \prime}=-\omega^{2} \chi_{\omega \alpha}$, which, indeed, has solutions $\left(\omega^{2} \in \mathbb{R}\right)$

$$
\chi_{\omega \alpha}= \begin{cases}\mu_{+} \sin \omega r+v_{+} \cos \omega r, & \omega^{2}>0 \\ \mu_{-} \mathrm{e}^{-|\omega| r}+v_{-} \mathrm{e}^{+|\omega| r}, & \omega^{2}<0 \\ \mu_{0} r+v_{0}, & \omega=0 .\end{cases}
$$

where the coefficients are to be verified by the boundary condition and integrability. The latter condition kills $v_{-}, \mu_{0}$ and $v_{0}$.

For $\omega^{2}>0$ the s-wave eigenfunction differs from the regular one $\sin \omega r / r$ and instead of $\left(\mu_{+}, v_{+}\right)$we conveniently write the radial part in the form

$$
u_{\omega \alpha}(r)=C\left(\frac{\sin \omega r}{r}+\tan \delta \frac{\cos \omega r}{r}\right)
$$

(with some mixture angle $\delta$ ), where the boundary condition at the origin yields

$$
\tan \delta=\frac{\omega}{4 \pi \alpha} .
$$

Normalizing our positive- and negative-frequency solutions with respect to the scalar product

$$
i \int d^{3} x \phi_{\omega}^{( \pm)^{*}} \overleftrightarrow{\partial_{t}} \phi_{\omega^{\prime}}^{( \pm)}= \pm \delta\left(\omega-\omega^{\prime}\right),
$$

or equivalently, in the form

$$
\int d^{3} x u_{\omega \alpha}^{*} u_{\omega^{\prime} \alpha}=\frac{\delta\left(\omega-\omega^{\prime}\right)}{2 \omega},
$$

we get $C=\cos \delta /(2 \pi \sqrt{\omega})$. Thus, the $s$-wave solution reads

$$
u_{\omega \alpha}(r)=\frac{1}{2 \pi r} \frac{\sin (\omega r+\delta)}{\sqrt{\omega}} .
$$

Let $\left\{u_{\omega l m}\right\}$ be the complete eigenfunction set of the free Hamiltonian. Note that

$$
\left.u_{\omega 00} \equiv u_{\omega \alpha}\right|_{\alpha=\infty} .
$$

By well-known results, if $\alpha \geqslant 0$, the self-adjoint operator $H_{0 \alpha}$ has no negative eigenvalues and it's complete set of generalized orthonormalized eigenfunctions consists of $\left\{u_{\omega l m}\right\}$ for $l>0$ and $u_{\omega, \alpha}(r)$ for $l=0$, where $u_{\omega l m}$ is

$$
u_{\omega l m}=v_{\omega l} Y_{l m}, \quad \dot{H}_{l} v_{\omega l}=\omega^{2} v_{\omega l} .
$$

By physical arguments this case describes a repulsive interaction.

If $-\infty<\alpha<0, H_{0 \alpha}$ has the single negative eigenvalue (a bound state),

$$
E_{\alpha}=-(4 \pi \alpha)^{2}, \quad-\infty<\alpha<0,
$$

with

$$
u_{0 \alpha}(r)=\frac{\sqrt{-\alpha}}{r} \mathrm{e}^{4 \pi \alpha r}
$$


as corresponding normalized eigenfunction. By physical arguments, this case describes an attractive interaction. Below we'll restrict our consideration by the repulsive case. In this case the two-point Hadamard's function takes the form:

$$
\begin{aligned}
D^{(1)}\left(x, x^{\prime}\right) & =\frac{1}{2}\left\langle\phi(x) \phi\left(x^{\prime}\right)+\phi\left(x^{\prime}\right) \phi(x)\right\rangle_{\mathrm{vac}}= \\
& =\operatorname{Re} \int_{0}^{\infty} d \omega e^{-i \omega\left(t-t^{\prime}\right)}\left[u_{\omega \alpha}(x) u_{\omega \alpha}^{*}\left(x^{\prime}\right)+\sum_{l=1}^{\infty} \sum_{m=-l}^{l} u_{\omega l m}(x) u_{\omega l m}^{*}\left(x^{\prime}\right)\right] .
\end{aligned}
$$

Thus we can represent the Hadamard function as a sum

$$
D^{(1)}\left(x, x^{\prime}\right)=D_{\infty}^{(1)}\left(x, x^{\prime}\right)+D_{\alpha}^{(1)}\left(x, x^{\prime}\right),
$$

where $D_{\infty}^{(1)}\left(x, x^{\prime}\right)$ denotes the free (flat space) Hadamard's elementary function,

$$
D_{\infty}^{(1)}\left(x, x^{\prime}\right)=\operatorname{Re} \int_{0}^{\infty} d \omega \mathrm{e}^{-i \omega\left(t-t^{\prime}\right)} \sum_{l=0}^{\infty} \sum_{m=-l}^{l} u_{\omega l m}(x) u_{\omega l m}^{*}\left(x^{\prime}\right) .
$$

In what follows $D_{\infty}^{(1)}$ is of interest for us just at its null level. It defines the vacuum state, since the renormalized values of this function, as well as of its derivatives, in the coincidence-points limit are equal to zero. Thus, we shall define $D_{\text {reg }}^{(1)}$ as a difference

$$
D_{\text {reg }}^{(1)}=D^{(1)}-D_{\infty}^{(1)}=D_{\alpha}^{(1)},
$$

and we obtain

$$
D_{\alpha}^{(1)}\left(x, x^{\prime}\right)=\operatorname{Re} \int_{0}^{\infty} d \omega \mathrm{e}^{-i \omega\left(t-t^{\prime}\right)}\left[u_{\omega \alpha}(x) u_{\omega \alpha}^{*}\left(x^{\prime}\right)-u_{\omega \infty}(x) u_{\omega \infty}^{*}\left(x^{\prime}\right)\right] .
$$

Rescaling of the integration variable $\omega=4 \pi \alpha z$ gives an eventual form

$$
D_{\alpha}^{(1)}\left(x, x^{\prime}\right)=\frac{1}{4 \pi^{2} r r^{\prime}} \int_{0}^{\infty} d z \frac{\cos \left[4 \pi \alpha z\left(t-t^{\prime}\right)\right]}{1+z^{2}}\left(\sin \left[4 \pi \alpha z\left(r+r^{\prime}\right)\right]+z \cos \left[4 \pi \alpha z\left(r+r^{\prime}\right)\right]\right) \text {. }
$$

\subsection{Field-Square VEV}

Taking the limit $t=t^{\prime}$ and $r^{\prime}=r$, we reexpress the result as

$$
\left\langle\phi^{2}(x)\right\rangle_{\text {ren }}=D_{\alpha}^{(1)}(x, x)=\frac{\mathcal{J}(8 \pi \alpha r)}{4 \pi^{2} r^{2}},
$$

in terms of introduced function

$$
\mathcal{J}(\beta) \equiv \int_{0}^{\infty} d z \frac{1}{1+z^{2}}(\sin \beta z+z \cos \beta z)
$$

This integral is to be computed in terms of special functions. Namely, we make use of the table integral [38] [f.3.723-1]:

$$
\int_{0}^{\infty} d z \frac{\sin \beta z}{1+z^{2}}=\frac{1}{2}\left[\mathrm{e}^{-\beta} \overline{\operatorname{Ei}}(\beta)-\mathrm{e}^{\beta} \operatorname{Ei}(-\beta)\right] \operatorname{sgn} \beta,
$$


where $\operatorname{Ei}(x)$ is the exponential integral function

$$
\operatorname{Ei}(x)=\int_{-\infty}^{x} \frac{\mathrm{e}^{t}}{t} d t
$$

well-defined for $x<0$ and analytically-continued to $\mathbb{C}$ except the cut $[0,+\infty)$, with $x=0$ to be the branching point. The values at the cut (on the main sheet) are $\overline{\operatorname{Ei}}(x)=$ $=[\operatorname{Ei}(x+i 0)+\operatorname{Ei}(x-i 0)] / 2$, and for any positive $x$ both Ei and $\overline{E i}$ in (36) are unambiguously presented by converging series

$$
\operatorname{Ei}(-x)=\gamma+\ln x+\sum_{k=1}^{\infty} \frac{(-x)^{k}}{k \cdot k !}, \quad \overline{\operatorname{Ei}}(x)=\gamma+\ln x+\sum_{k=1}^{\infty} \frac{x^{k}}{k \cdot k !},
$$

where $\gamma \approx 0.5772$ is the Euler-Mascheroni constant. Differentiating (36) with respect to $\beta$ one obtains

$$
\int_{0}^{\infty} d z \frac{z \cos \beta z}{1+z^{2}}=-\frac{1}{2}\left[\mathrm{e}^{-\beta} \overline{\operatorname{Ei}}(\beta)+\mathrm{e}^{\beta} \operatorname{Ei}(-\beta)\right],
$$

Combining (37) with (36), the total integral equals

$$
\mathcal{J}(\beta)= \begin{cases}-\mathrm{e}^{\beta} \operatorname{Ei}(-\beta), & \beta>0 ; \\ -\mathrm{e}^{-\beta} \overline{\operatorname{Ei}}(\beta), & \beta<0 .\end{cases}
$$

In order to don't deal with the multivalued function $\mathrm{Ei}$, for positive argument $(\beta)$, which is of our interest here, the Ei-function on the main sheet reduces to the modified (not analytic) exponential integral function $E_{1}: 6$

$$
-\operatorname{Ei}(-x)=\int_{x}^{\infty} \frac{\mathrm{e}^{-t}}{t} d t \equiv E_{1}(x), \quad x>0 .
$$

The vacuum field square then reads

$$
\left\langle\phi^{2}(r)\right\rangle_{\text {ren }}=\frac{\mathrm{e}^{8 \pi \alpha r} E_{1}(8 \pi \alpha r)}{4 \pi^{2} r^{2}} .
$$

The plot of $\left\langle\phi^{2}(r)\right\rangle_{\text {ren }}$ for different values of $\alpha$ is presented on the Figure 1. As it seen, each curve rapidly decays. In order to reveal some characteristic regimes of them, we will use the logarithmic coordinates (Figure 4 below).

Asymptotic regimes. The limit $\alpha \rightarrow+\infty$ corresponds to flat space with no potential. Therefore the regime $r \gg \alpha^{-1}$ corresponds to the case where the interaction is weak.

For the exponential integral the asymptotic representation [38]

$$
\mathrm{e}^{x} E_{1}(x)=\frac{1}{x} \sum_{k=0}^{\infty} \frac{(-1)^{k} k !}{x^{k}}, \quad x \gg 1
$$

is available, thus at large distances $\left(r \gg \alpha^{-1}\right)$ the $\operatorname{VEV}\left\langle\phi^{2}\right\rangle_{\text {ren }}$ reads

$$
\left\langle\phi^{2}(r)\right\rangle_{\text {ren }}=\frac{1}{32 \pi^{3} \alpha r^{3}}\left[1+\mathcal{O}\left(\beta^{-1}\right)\right] .
$$

\footnotetext{
6 Notice, we use the definitions of [39]. Not to be confused with the 1st Euler's polynomial
} 


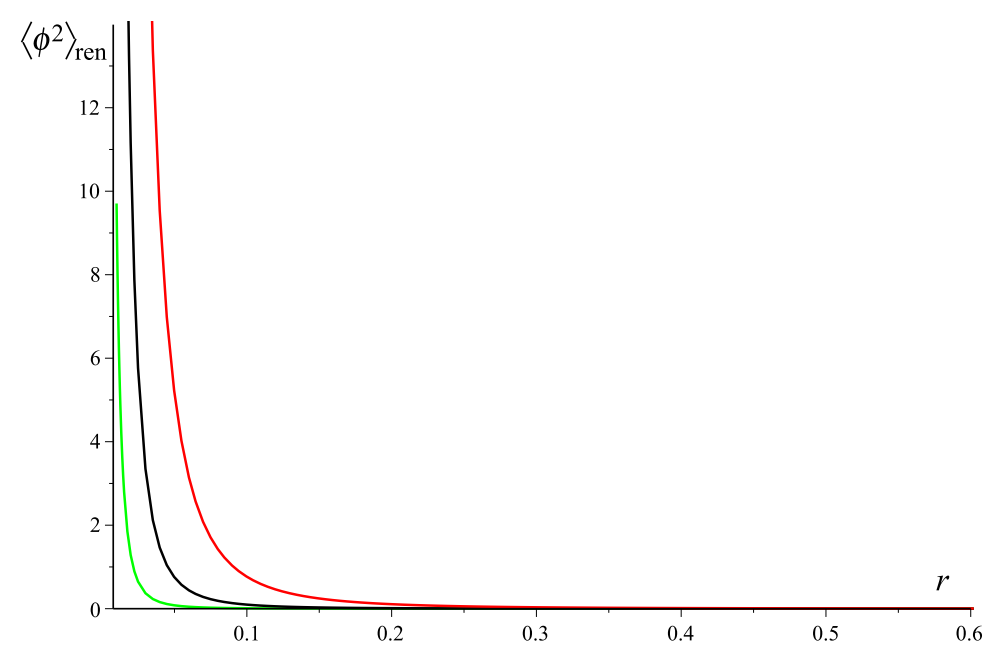

Figure 1. Plots of $\left\langle\phi^{2}\right\rangle_{\text {ren }}$ as function of $r$ for $\alpha=1$ (red), $\alpha=10$ (black) and $\alpha=100$ (green).

A necessary note should be done: the expansion (40) is asymptotic, but the infinite series obviously diverges for any $x$. Nevertheless, for fixed value $x$ the highest precision is achieved for the partial sum with $k_{\max }(x)=[x]$ instead of infinity in (40):

$$
\left\langle\phi^{2}(r)\right\rangle_{\text {ren }}=\frac{1}{32 \pi^{3} \alpha r^{3}} \sum_{k=0}^{[8 \pi \alpha r]} \frac{(-1)^{k} k !}{(8 \pi \alpha r)^{k}} .
$$

If truncate the series at this recommended value, the relative error for $\left\langle\phi^{2}(r)\right\rangle_{\text {ren }}$ is $\mathcal{O}\left(\sqrt{4 \pi^{2} \alpha r} \mathrm{e}^{-8 \pi \alpha r}\right)$. We can not approach the true value more closely, but such an error exponentially decreases. Thereby we consider it as a good approximation.

The different approximations to $\left\langle\phi^{2}(r)\right\rangle_{\text {ren }}$ by the asymptotic Formula (40) are presented on the Figure 2.

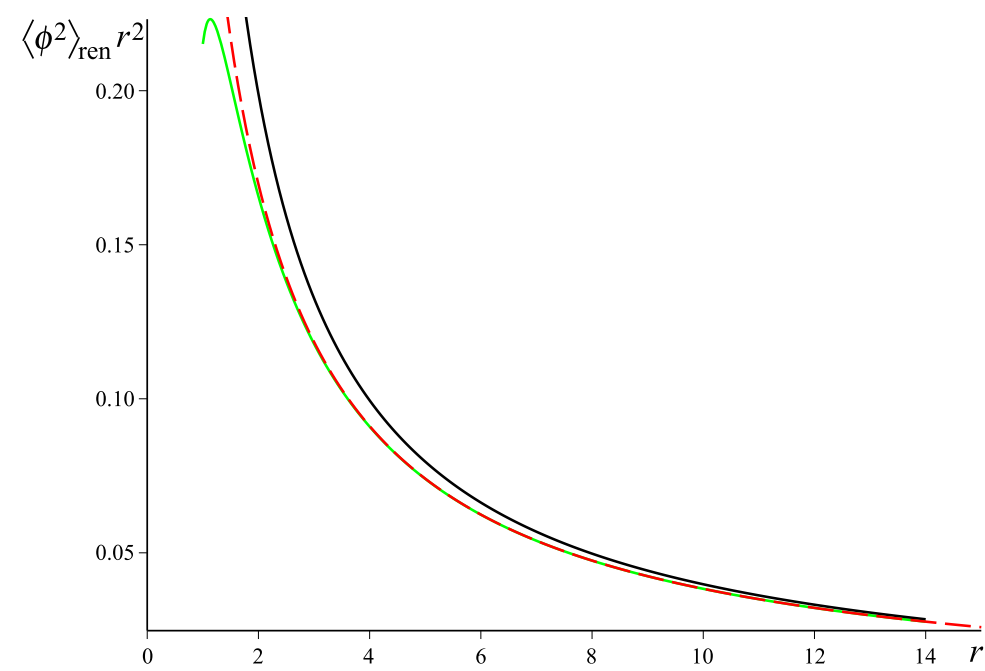

Figure 2. Plots of $\left\langle\phi^{2}\right\rangle_{\text {ren }}$ (times $r^{2}$ ) for $\alpha=0.1$ : computed exactly (by Equation (39), red dashed curve), for the main asymptotic term $\left(k_{\max }=0\right.$, by Equation (41), black curve) and for $k_{\max }=3$ (in Equation (40), green).

The difference between the best approximation (by Equation (42)) and the worst one (by Equation (41)) is shown in Figure 3. In order to reveal the difference we plot the small domain and for small value of $\alpha$ in doubly logarithmic mode. The miser drawback of the usage of the $r$-dependent truncation for the best approximation consists in the obvious fact 
that being plotted on some domain, the curve is not continuous. Indeed, when the product $8 \pi \alpha r$ reaches an integer, the number of retained terms shifts by one and the plot becomes piecewise (Figure 3 , the blue curve has discontinuities at $\ln r \approx 1.903$ and 1.217).

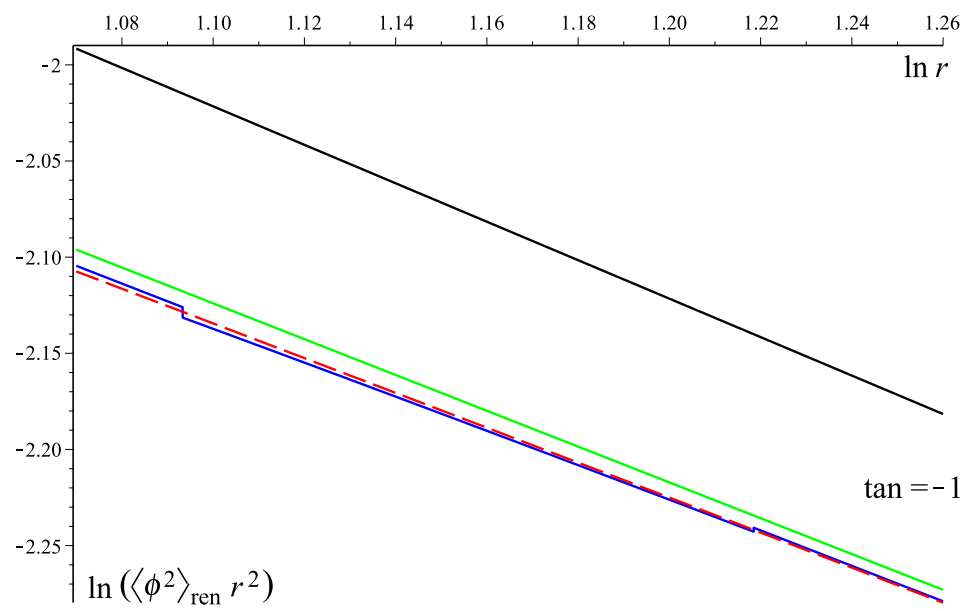

Figure 3. Plots of $\ln \left(r^{2}\left\langle\phi^{2}\right\rangle_{\text {ren }}\right)$ versus $\ln r$ for $\alpha=0.1$, computed exactly (by Equation (39), red dashed curve), for the main asymptotic term ( $k_{\max }=0$, by Equation (41), black curve), for $k_{\max }=3$ (in Equation (40), green) and for the $r$-truncated series (by Equation (42), blue). The plotting domain corresponds to $\alpha r=0.29 \ldots 0.35$. For higher $r, \alpha$ and more wide range the curves become closer to each other.

For the opposite case of small distances $\left(r \ll \alpha^{-1}\right)$ one uses the series [38]

$$
E_{1}(x)=|\ln x|-\gamma-\sum_{k=1}^{\infty} \frac{(-x)^{k}}{k \cdot k !}, \quad 0<x \ll 1 .
$$

Thus the vacuum averaged $\left\langle\phi^{2}\right\rangle_{\text {ren }}$ takes the form

$$
\left\langle\phi^{2}(r)\right\rangle_{\text {ren }}=\frac{1}{4 \pi^{2} r^{2}}\left(-\ln \left(8 \pi \mathrm{e}^{\gamma} \alpha r\right)+\mathcal{O}(\alpha r|\ln \alpha r|)\right) .
$$

The behavior of $\left\langle\phi^{2}\right\rangle_{\text {ren }}$ with respect to asymptotic regimes is shown in Figure 4 in doubly logarithmic mode.

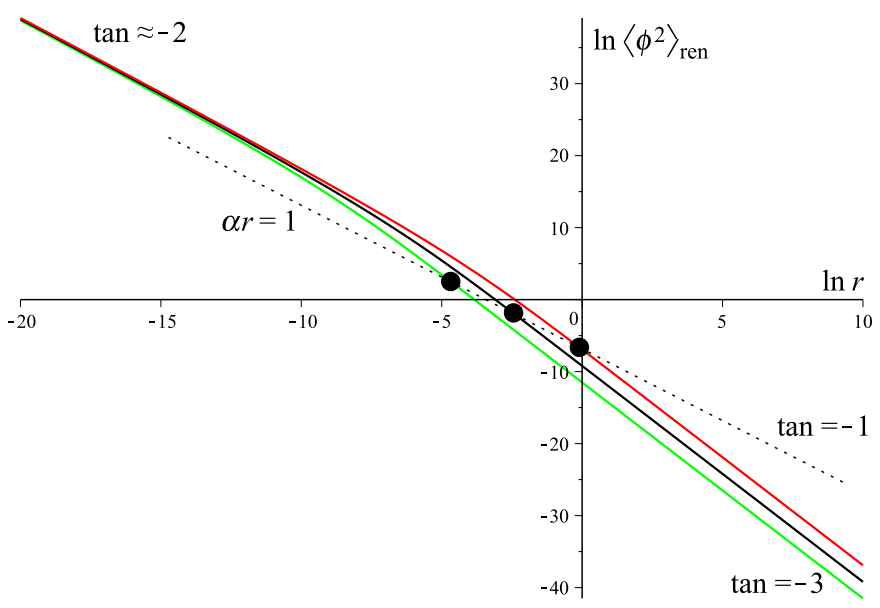

Figure 4. Plots of $\ln \left\langle\phi^{2}\right\rangle_{\text {ren }}$ versus $\ln r$ for $\alpha=1$ (red), $\alpha=10$ (black) and $\alpha=100$ (green). Dots on each curve correspond to the value $r=\alpha^{-1}$ and distinguish two characteristic regimes. 
It contains two characteristic regimes with tangents approximately equal to -2 (that corresponds to $r \ll \alpha^{-1}$ ) and -3 (respectively, to $r \gg \alpha^{-1}$ ), and some transition area across $r=(8 \pi \alpha)^{-1}$.

\subsection{Vacuum Energy-Momentum Tensor}

The vacuum energy-momentum tensor for a massless field in a flat space-time may be determined by the application of the differential operator (14) to the constructed Green's function (35), i.e., already in the regularized form. We notice, that for $r, r^{\prime}>0 D_{\alpha}^{(1)}\left(x, x^{\prime}\right)$ satisfies the free d'Alembert equation:

$$
\square_{x} D_{\alpha}^{(1)}\left(x, x^{\prime}\right)=0=\square_{x^{\prime}} D_{\alpha}^{(1)}\left(x, x^{\prime}\right)
$$

and thus the last term in (14) may be omitted:

$$
\left\langle T_{\mu}^{v}\right\rangle_{\text {ren }}=\lim _{x^{\prime} \rightarrow x}\left[(1-2 \xi) \partial^{v} \partial_{\mu}^{\prime}-\left(\frac{1}{2}-2 \xi\right) \delta_{\mu}^{v} \partial^{\lambda} \partial_{\lambda}^{\prime}-\xi\left(\nabla^{v} \partial_{\mu}+\nabla^{\prime v} \partial_{\mu}^{\prime}\right)\right] D_{\alpha}^{(1)}\left(x, x^{\prime}\right) .
$$

The covariant derivative here is essential, since we use the curvilinear (spherical) coordinates, and the Christoffel symbol do not vanish. ${ }^{7}$

Computing the derivatives over $t\left(t^{\prime}\right)$ and $r\left(r^{\prime}\right)$, and so on, the integral (35) accumulates $z$ in numerator and becomes UV-divergent. At the other hand, we can not differentiate $\mathcal{J}(\beta)$ directly since it is defined already with the coincident points. Since $D_{\alpha}^{(1)}\left(x, x^{\prime}\right)$ is angular-independent, we need to regularize derivatives over $r\left(r^{\prime}\right)$ and $t\left(t^{\prime}\right)$, then setting the limits $t=t^{\prime}, r=r^{\prime}$.

Start with the spatial derivatives: we separate the integral in (35) as

$$
\begin{aligned}
& D_{\alpha}^{(1)}\left(x, x^{\prime}\right)=\frac{I\left(t-t^{\prime}, r+r^{\prime}\right)}{4 \pi^{2} r r^{\prime}} \\
& I \equiv \int_{0}^{\infty} d z \frac{\cos \left[4 \pi \alpha z\left(t-t^{\prime}\right)\right]}{1+z^{2}}\left(\sin \left[4 \pi \alpha z\left(r+r^{\prime}\right)\right]+z \cos \left[4 \pi \alpha z\left(r+r^{\prime}\right)\right]\right),
\end{aligned}
$$

which in the coincident-point limit becomes:

$$
I(0,2 r)=\mathcal{J}(8 \pi \alpha r) .
$$

Both radial coordinates enter in the integrand as a sum, thus

$$
\frac{\partial I\left(t-t^{\prime}, r+r^{\prime}\right)}{\partial r}=\frac{\partial I\left(t-t^{\prime}, r+r^{\prime}\right)}{\partial r^{\prime}}=\frac{\partial I\left(t-t^{\prime}, r+r^{\prime}\right)}{\partial\left(r+r^{\prime}\right)} .
$$

Therefore if to introduce the new single variable $R \equiv r+r^{\prime}$, then one notices that the computation of the limit $r=r^{\prime}$ does not change the total independent $R$, while the limit $t=t^{\prime}$ does not involve the differentiation variable $R$ and just transforms $I$ to $\mathcal{J}$. In other words, the two operations-differentiation over $R$ and the coincident-point limit-commute. So one can differentiate (46) in the form

$$
I(t-t, R)=\mathcal{J}(4 \pi \alpha R)
$$

to obtain the correspondence rules

$$
\left.\frac{\partial I\left(t-t^{\prime}, r+r^{\prime}\right)}{\partial r}\right|_{x^{\prime}=x}=\frac{1}{2} \frac{\partial \mathcal{J}(8 \pi \alpha r)}{\partial r},\left.\quad \frac{\partial^{2} I\left(t-t^{\prime}, r+r^{\prime}\right)}{\partial r^{2}}\right|_{x^{\prime}=x}=\frac{1}{4} \frac{\partial^{2} \mathcal{J}(8 \pi \alpha r)}{\partial r^{2}} .
$$

7 Namely, the actual non-vanishing Christoffels for us will be $\Gamma_{\theta \theta}^{r}=-r$ and $\Gamma_{\varphi \varphi}^{r}=-r \sin ^{2} \theta$. 
The first time-derivatives are proportional to $\sin \left(t-t^{\prime}\right)$ and thus vanish in the limit:

$$
\left.\frac{\partial I\left(t-t^{\prime}, r+r^{\prime}\right)}{\partial t}\right|_{t^{\prime}=t}=-\left.\frac{\partial I\left(t-t^{\prime}, r+r^{\prime}\right)}{\partial t^{\prime}}\right|_{t^{\prime}=t}=0 .
$$

For the second time-derivatives we note that the constituents of the integrand of I satisfy

$$
\left(\frac{\partial^{2}}{\partial t^{2}}-\frac{\partial^{2}}{\partial r^{2}}\right)\left\{\begin{array}{l}
\cos \left[c\left(t-t^{\prime}\right)\right] \sin \left[c\left(r+r^{\prime}\right)\right] \\
\cos \left[c\left(t-t^{\prime}\right)\right] \cos \left[c\left(r+r^{\prime}\right)\right]
\end{array}\right\}=0,
$$

$(c=4 \pi \alpha z)$, and the same for $\left(\partial / \partial t \cdot \partial / \partial t^{\prime}+\partial / \partial r \cdot \partial / \partial r^{\prime}\right)$. Since both $r\left(r^{\prime}\right)$ and $t\left(t^{\prime}\right)$ are not the integration variable, the same properties hold for the total integral. Thus we convert time-derivatives into the $r$-derivative and reduce the problem to the already solved.

Thereby, we reduce all valuable derivatives in (45) into the form of derivatives of $\mathcal{J}(\beta)$. Namely, in the limit $x \rightarrow x^{\prime}$, apart from $\mathcal{J}(\beta)$, one encounters the derivatives $\mathcal{J}^{\prime}(\beta)$ and $\mathcal{J}^{\prime \prime}(\beta)$. To simplify the routine computations, we define the convenient combinations:

$$
\mathcal{J}_{1}(\beta) \equiv \beta \frac{d \mathcal{J}(\beta)}{d \beta}, \quad \mathcal{J}_{2}(\beta) \equiv \beta^{2} \frac{d^{2} \mathcal{J}(\beta)}{d \beta^{2}} .
$$

With help of the them the straightforward calculation leads to the following result for the components of the renormalized energy-momentum tensor in spherical coordinates:

$$
\begin{aligned}
\left\langle T_{t}^{t}\right\rangle_{\text {ren }} & =\frac{1}{4 \pi^{2} r^{4}}\left[\left(\frac{1}{2}-2 \xi\right) \mathcal{J}(8 \pi \alpha r)+\left(2 \xi-\frac{1}{2}\right) \mathcal{J}_{1}(8 \pi \alpha r)-\xi \mathcal{J}_{2}(8 \pi \alpha r)\right] \\
\left\langle T_{r}^{r}\right\rangle_{\text {ren }} & =\frac{1}{4 \pi^{2} r^{4}}\left[\left(4 \xi-\frac{1}{2}\right) \mathcal{J}(8 \pi \alpha r)+\left(\frac{1}{2}-2 \xi\right) \mathcal{J}_{1}(8 \pi \alpha r)\right] \\
\left\langle T_{\theta}^{\theta}\right\rangle_{\text {ren }} & =\frac{1}{4 \pi^{2} r^{4}}\left[\left(\frac{1}{2}-4 \xi\right) \mathcal{J}(8 \pi \alpha r)+\left(3 \xi-\frac{1}{2}\right) \mathcal{J}_{1}(8 \pi \alpha r)+\left(\frac{1}{4}-\xi\right) \mathcal{J}_{2}(8 \pi \alpha r)\right] \\
& =\left\langle T_{\varphi}^{\varphi}\right\rangle_{\text {ren }} \\
\left\langle T_{r}^{t}\right\rangle_{\text {ren }} & =0
\end{aligned}
$$

where the direct differentiation of (38) yields

$$
\mathcal{J}_{1}(\beta)=\beta \mathcal{J}(\beta)-1, \quad \mathcal{J}_{2}(\beta)=\beta^{2} \mathcal{J}(\beta)-\beta+1 .
$$

From the diagonal components we find that the trace equals

$$
\left\langle T_{\mu}^{\mu}\right\rangle_{\text {ren }}=(1-6 \xi) \frac{2 \mathcal{J}(8 \pi \alpha r)-2 \mathcal{J}_{1}(8 \pi \alpha r)+\mathcal{J}_{2}(8 \pi \alpha r)}{8 \pi^{2} r^{4}} .
$$

Thus, for the conformal, $\xi=1 / 6$, field the trace vanishes, in agreement with the generic expression for the conformal anomaly in the flat background $[33,36] .8$

Conservation law. After some straightforward computation one can check that the renormalized energy-momentum tensor is conserved:

$$
\nabla_{\nu}\left\langle T_{\mu}^{v}\right\rangle_{\text {ren }}=0 .
$$

More concretely, the covariant divergence of the symmetric tensor,

$$
\nabla_{\nu}\left\langle T_{\mu}^{v}\right\rangle_{\text {ren }}=|g|^{-1 / 2} \partial_{\nu}\left(|g|^{1 / 2}\left\langle T_{\mu}^{v}\right\rangle_{\text {ren }}\right)-\frac{1}{2}\left\langle T_{\rho}^{v}\right\rangle_{\text {ren }} g^{\lambda \rho} \partial_{\mu} g_{\nu \lambda},
$$

\footnotetext{
8 Here we are not interested in the problem whether the potential, being an energy source, produces own gravitational field.
} 
in our static case with the dependence upon the radial coordinate only, is trivial for $\mu=t, \theta, \varphi$. The conservation of $\mu=r$ component reduces to

$$
\beta R^{\prime}(\beta)+2[R(\beta)+\Phi(\beta)]=0,
$$

where $R \equiv 4 \pi^{2} r^{4} T_{r}^{r}, \Phi \equiv 4 \pi^{2} r^{4} T_{\varphi}^{\varphi}$ (the square parentheses in Equations (47) and (48)). To satisfy (52), one easily verifies that the two following necessary conditions hold: (i) $R$ does not contain $\mathcal{J}_{2}$, and (ii) $(R+\Phi)$ does not contain $\mathcal{J}$ directly. It follows from the fact that $\mathcal{J}$, $\mathcal{J}_{1}$ and $\mathcal{J}_{2}$ have different asymptotic behavior at infinity. in (51).

Plugging (47) and (48) into (52) one verifies that the condition does hold, resulting

Asymptotic regimes. For large values of its argument the condition $\mathcal{J}(x)=1 / x+$ $+\mathcal{O}\left(x^{-2}\right)$ for $\beta \gg 1$ reduces to

$$
\left\langle T_{\mu}^{v}(t, r, \theta, \varphi)\right\rangle_{\text {ren }}=-\frac{3}{16 \alpha \pi^{3} r^{5}}\left(\xi-\frac{1}{6}\right) \operatorname{diag}\left(1,-1, \frac{3}{2}, \frac{3}{2}\right)\left[1+\mathcal{O}\left(\frac{1}{\alpha r}\right)\right],
$$

where we imply that $|\xi|$ does not take large values.

We notice, that the exact result for EMT does not vanish for the conformal coupling, in contrast with the (53). Now we can get the corresponding asymptotic for this case:

$$
\left\langle\tilde{T}_{\mu}^{v}(t, r, \theta, \varphi)\right\rangle_{\text {ren }}=\frac{1}{512 \alpha^{2} \pi^{4} r^{6}} \operatorname{diag}\left(1, \frac{1}{3},-\frac{2}{3},-\frac{2}{3}\right)\left[1+\mathcal{O}\left(\frac{1}{\alpha r}\right)\right], \quad \xi=1 / 6 .
$$

Thus the renormalized EMT of the conformal field decays faster. The trace, indeed, vanishes. For the estimates of approximation of EMT by the asymptotic series, all the arguments presented above for $\left\langle\phi^{2}\right\rangle$, are still valid.

In fact, the expression (54) represents an asymptotic to the corresponding exact tensor $\left\langle\tilde{T}_{\mu}^{v}\right\rangle \equiv\left\langle\tilde{T}_{\mu}^{v}\right\rangle_{\mathcal{\xi}=1 / 6^{\prime}}$, so that the following splitting holds:

$$
\left\langle T_{\mu}^{v}\right\rangle_{\text {ren }}=\tilde{T}_{\mu}^{v}+\left(\xi-\frac{1}{6}\right) \bar{T}_{\mu}^{v}(\xi),
$$

thus $\tilde{T}_{\mu}^{v}$ and $\bar{T}_{\mu}^{v}(\xi)$ may be regarded as conformal and "anti-conformal" parts of the renormalized EMT, respectively. From (50) we conclude that

$$
\tilde{T}_{\varphi}^{\varphi}=-\frac{\tilde{T}_{t}^{t}+\tilde{T}_{r}^{r}}{2} .
$$

The plot of the non-zero components of the conformal part $\left(\tilde{T}_{\mu}^{v}\right)$ is given on the Figure 5.

In the limit $r \rightarrow \infty$ the conformal tensor scales as $\tilde{T}_{\mu}^{v} \propto r^{-6}$ while the "anti-conformal" one as $\bar{T}_{\mu}^{v} \propto r^{-5}$. In what follows $\bar{T}_{\mu}^{v}$ dominates (for $\xi \neq 1 / 6$ ) and thus its asymptotic behavior is determined by Equation (53). The plot of the non-zero components of the "anti-conformal" part $\left(\bar{T}_{\mu}^{v}(\xi)\right)$ is presented in Figure 6.

For the opposite case, $\beta \ll 1$, we use expansion (43). Since the derivatives of $\mathcal{J}$ contain extra powers of $r$, by virtue of Equation (49), for $\beta \ll 1$ the behavior of the renormalized EMT components is governed by the $\mathcal{J}$-coefficients. Retaining the two basic terms, we get:

$$
\begin{aligned}
& \left\langle T_{\mu}^{v}(t, r, \theta, \varphi)\right\rangle_{\text {ren }}=\frac{1}{4 \pi^{2} r^{4}}\left[\operatorname{diag}\left(2 \xi-\frac{1}{2}, \frac{1}{2}-4 \xi, 4 \xi-\frac{1}{2}, 4 \xi-\frac{1}{2}\right) \ln 8 \pi \alpha r+\right. \\
& \left.+\operatorname{diag}\left(\frac{1-\gamma}{2}+\xi(2 \gamma-3), \frac{\gamma-1}{2}+\xi(2-4 \gamma), \frac{3-2 \gamma}{4}+4 \xi(\gamma-1), \frac{3-2 \gamma}{4}+4 \xi(\gamma-1)\right)\right],
\end{aligned}
$$

plus the remainder of order $\mathcal{O}\left(\alpha \ln (\alpha r) / r^{3}\right)$. 


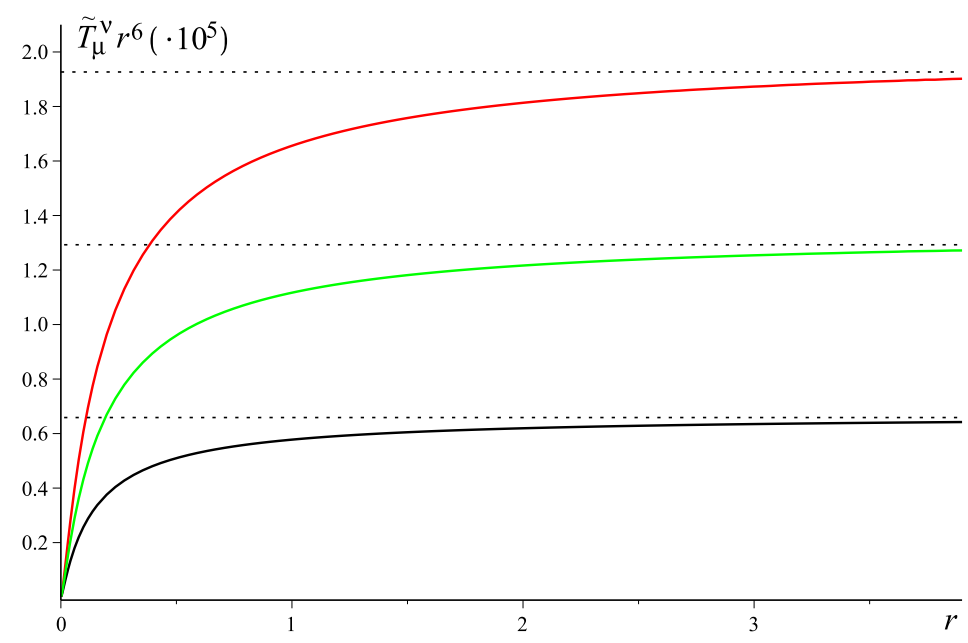

Figure 5. Plots of the conformal part of $\left\langle T_{\mu}^{v}\right\rangle_{\text {ren }}$ (normalized by the overall factor $r^{6}$ ) for $\alpha=1: \tilde{T}_{t}^{t}$ (red), $\tilde{T}_{r}^{r}$ (black), and $-\tilde{T}_{\varphi}^{\varphi}$ (green). Horizontal asymptotes at $r \rightarrow \infty$ and their ratio correspond to Equation (54).

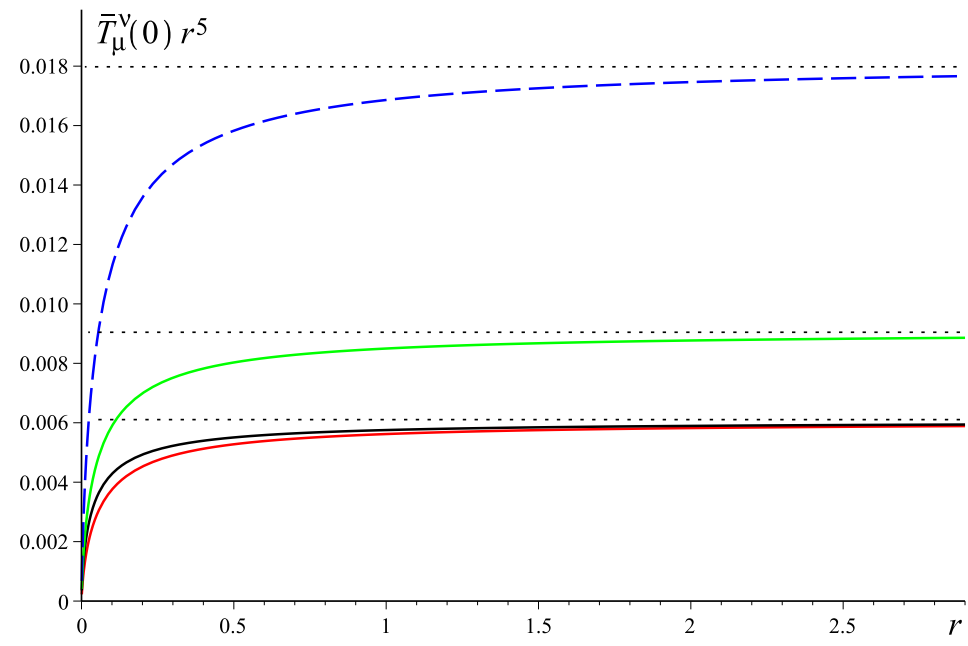

Figure 6. Plots of the "anti-conformal" tensor $\bar{T}_{\mu}^{v}(\xi)$ (normalized by the overall factor $r^{5}$ ) for minimal coupling $(\xi=0)$ and $\alpha=1:-\bar{T}_{t}^{t}$ (red), $+\bar{T}_{r}^{r}$ (black), and $-\bar{T}_{\varphi}^{\varphi}$ (green) and for the trace $-\operatorname{Sp} \bar{T}$ (blue dashed). Horizontal asymptotes at $r \rightarrow \infty$ and their ratio correspond to Equation (53). The ratio Sp $\bar{T} / \bar{T}_{t}^{t}$ asymptotically corresponds to Equation (20).

The behavior of the renormalized vacuum energy density for the total EMT $\left\langle T_{\mu}^{v}\right\rangle_{\text {ren }}$ and its constituents $\left(\tilde{T}_{\mu}^{v}\right.$ and $\left.\bar{T}_{\mu}^{v}(\xi)\right)$ is shown in Figure 7 .

To summarize, the problem of vacuum polarization of the massless scalar field in the external zero-width barrier may be solved exactly for the case of repulsive potential, by means of the Laplacian's self-adjoint extension. The final answer for the renormalized vacuum $\left\langle\phi^{2}\right\rangle$ and $\left\langle T_{\mu \nu}\right\rangle$ is expressed in terms of the exponential integral $E_{1}$. The extension parameter $\alpha$ and the observation point $r$ enter the formulae in the dimensionless combination $\beta=8 \pi \alpha r$. Therefore from the behavior of $E_{1}$ (pure mathematical, not related to any physical problem) there exists a natural domain $\beta \simeq 1$ which distinguishes asymptotic regimes of the problem at hand. At fixed $r$, the "almost-free-Hamiltonian" regime is get for the extremely large extension parameters; in terms of the exponential integral it corresponds to the large- $\beta$ asymptotic regime $(\beta \gg 1)$. In turn, for fixed extension parameter $\alpha$, such a regime corresponds to the large distances from the source $\left(r \gg \alpha^{-1}\right)$. In other words, the extension parameter $\alpha$ plays the role of the inverse "characteristic radius" of the point-like interaction. Thus (41) and (53) correspond to the weak-field approximation. 


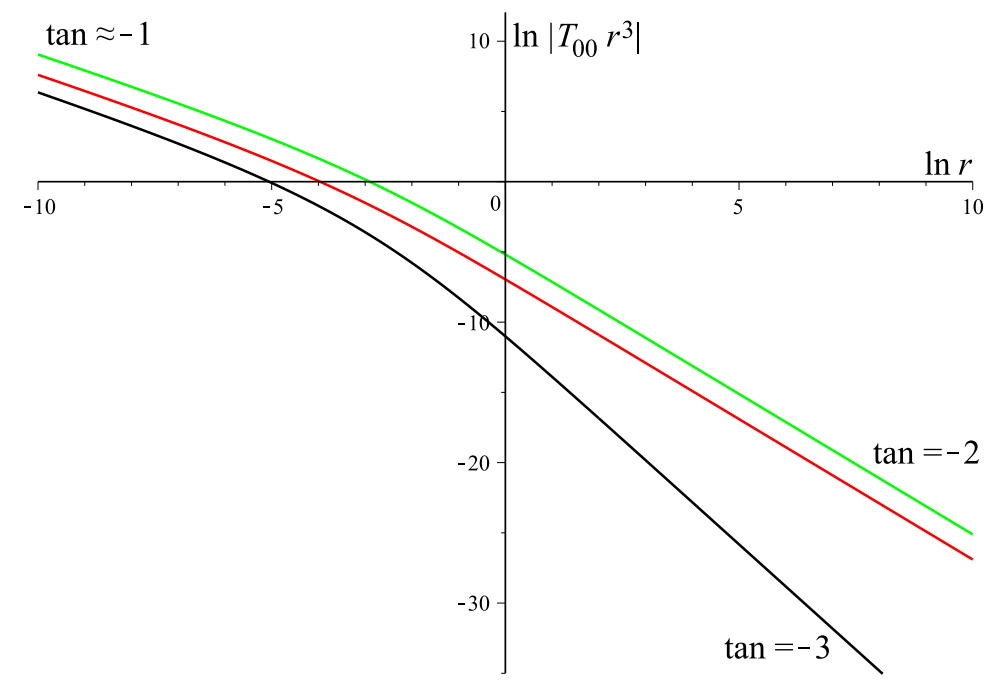

Figure 7. Plots of the vacuum energy density (times overall factor $r^{3}$ ) in doubly logarithmic mode $(\alpha=1)$ : for the total EMT $\left\langle T_{00}\right\rangle_{\text {ren }}\left(\xi=0\right.$, red), for conformal tensor $\tilde{T}_{00}$ (black), and for "anticonformal" tensor $-\bar{T}_{00}(\xi)(\xi=0$, green).

The exact EMT trace is proportional to the factor $(\xi-1 / 6)$ and vanishes for the conformal coupling $\xi=1 / 6$, in accordance with the conformal anomaly. The full answer for the total EMT is not proportional to the factor $(\xi-1 / 6)$ and thus does not vanish for the conformal field. Results for the renormalized EMT coincide with the ones obtained by the heat kernel technique in [10].

\section{Discussion}

We have considered a problem of the vacuum polarization of a scalar field in the presence of zero-width potential within two different frameworks. The first considers the $\delta$ like potential as a distributional limit of potentials with compact support tending the width to zero and fixing the source's field strength; it works in the weak-field approximation. The second implies the heuristically written operator $-\Delta+\lambda \delta$ as a self-adjoint extension of the free Laplacian on the space with extracted point; the results obtained here, are nonperturbative. The results of both approaches are expressed in terms of "own" couplings: via delta-field coupling $\lambda$ in Section 2 and via the SAE-parameter $\alpha$ in Section 3 .

Making the comparison of the renormalized $\left\langle\phi^{2}(r)\right\rangle_{\text {ren }}$ in (13) (which is valid at $r \gg \lambda$ ) and the corresponding weak-field asymptotics (41) from Section 3 , one deduces that the relation of constants (if it does take place!) is given by

$$
\alpha=-1 / \lambda .
$$

Comparing the corresponding results for the vacuum energy density (Formula (23) versus (53)), as well as for the spatial components of the renormalized EMT, we arrive at the same conclusion.

Thus a question arises of whether one can consider both approaches as adequate descriptions of the same zero-width potential, if in both cases the absence of bound states is identified with the positive values of the corresponding coupling. One might suppose that the solution lies in the necessity of renormalization of the "bare" coupling $\lambda$.

In fact, let the perturbed operator $H_{\lambda}=-\Delta+\lambda \delta$ has an eigenfunction $u_{\omega}(\boldsymbol{x})$, which corresponds to the continuous spectrum. The general solution for the central field is a superposition of functions $u_{\omega}(\boldsymbol{x})=R_{l}(r) Y_{l m}(\vartheta, \varphi)$. Integrate an eigenfunction equation 
$H_{\lambda} u_{\omega}(\boldsymbol{x})=\omega^{2} u_{\omega}(\boldsymbol{x})$ over a ball $B_{\varepsilon}$ with radius $\varepsilon$ centered at $\boldsymbol{x}=\mathbf{0}$. Applying the Gauß theorem, one obtains:

$$
-\int_{\partial B_{\varepsilon}} \nabla u_{\omega} d \boldsymbol{\Sigma}+\lambda u_{\omega}(\mathbf{0})=\frac{4 \pi \varepsilon^{3} \omega^{2}}{3}\left\langle u_{\omega}(\boldsymbol{x})\right\rangle_{\varepsilon},
$$

where $\left\langle u_{\omega}(\boldsymbol{x})\right\rangle_{\varepsilon}$ stands for the average value of $u_{\omega}$ over a ball. In what follows

$$
-4 \pi \varepsilon^{2}\left\langle Y_{l m}\right\rangle R_{l}^{\prime}(\varepsilon)+\lambda u_{\omega}(0)=\frac{4 \pi \varepsilon^{3} \omega^{2}}{3}\left\langle Y_{l m}\right\rangle R_{l}(0)[1+\mathcal{O}(\varepsilon)]
$$

where $\left\langle Y_{l m}\right\rangle$ is a mean value of $Y_{l m}$ over $S^{2}$. Admitting the extension of the definitiondomain onto a class of (locally) square-integrable functions which go like $\sim r^{-1}$ as $r \rightarrow 0^{+}$and taking into account the well-known radial-function behavior $R_{l} \sim r^{l}$, we convince ourselves that the extension affects on the s-wave only:

$$
R_{0}(r)=\frac{f_{-1}}{r}+f_{0}+f_{1} r+\ldots ;
$$

indeed, a shift of the power of $r$ by one less does not make irregular the functions with $l \geqslant 1$. Then for the $s$-state one has $Y_{00}=1$ and $u_{\omega}=R_{0}$ is a spherically-symmetric function. Tending $\varepsilon \rightarrow 0^{+}$, in the limit we obtain a constraint

$$
4 \pi f_{-1}+\lambda f_{0}=0 .
$$

In fact, we have reproduced here the same ideology, which is of use in the SAE approach, for the perturbed Hamiltonian $H_{\lambda}$. Then in the assumption of the equivalence (correspondence) of both approaches in Sections 2 and $3{ }^{9}$ we have to identify the $s$-wave $u_{\omega}$ of the perturbed Hamiltonian with the $s$-wave $u_{\omega \alpha}$ of the free-Hamiltonian's SAE (33). Hence we write the same coefficients $f_{-1}, f_{0}$ for both radial functions. In terms of the SAE parameter of the free Laplacian with extracted origin, from Equation (31) it follows

$$
4 \pi \alpha \lim _{r \rightarrow 0^{+}} r u_{\alpha}(r)=\lim _{r \rightarrow 0^{+}}\left(u_{\alpha}+r u_{\alpha}^{\prime}\right), \quad f_{0}=4 \pi \alpha f_{-1} .
$$

Thus, we conclude the relation $\alpha \lambda=-1$, which coincides with (56) but the absence of bound states (which is considered as repulsion) should correspond to both $\lambda>0$ and $\alpha>0$ ! The resolution consists in the fact that in the formal integration of the eigenfunction equation the term $\lambda \delta$ yields $\lambda f_{0}$ before the application of SAE. If to do it with account of (57), it appears an addendum $\lambda f_{-1} / \epsilon$, where $\epsilon$ tends to $0^{+}$and has no relation with the radius of an integration domain $B_{\varepsilon} .{ }^{10}$ An account of this infinite contribution indicates on the necessity of renormalization of coupling $\lambda$ :

$$
\frac{1}{\lambda_{\text {ren }}}=\frac{1}{\lambda}+\frac{1}{4 \pi \epsilon} .
$$

The need of this renormalization at the QM level was discussed in several works. The relation of "bare" coupling with the SAE parameter was considered in [17,18,40]. In [25] it was pointed out that such a renormalization eliminates the leading divergence in the effective action.

9 What represents a goal of our issue.

10 The parameter $\epsilon$ appears from reexpressing (21) as

$$
\delta^{3}(x)=\frac{\delta_{+}(r)}{4 \pi r^{2}}=\lim _{\epsilon \rightarrow 0^{+}} \frac{1}{4 \pi} \frac{\delta(r-\epsilon)}{r^{2}} .
$$


Hence it is natural to suppose that the resolution of the sign paradox consists in the substitution of the "bare" $\lambda$ by $\lambda_{\text {ren }}$. In terms of the renormalized coupling, the relation with the SAE parameter is given by $\alpha=-\lambda_{\text {ren }}^{-1}$.

Though no renormalization was necessary within both computations, we must do it now in order to achieve self-consistent results and adequate interpretation of them. The main achievement of the work is a computation of non-perturbative effects with SAE, where the SAE-parameter is to be connected with the renormalized strength coupling. In what follows, the coupling $\lambda$ in the results of Section 2 should also be regarded in the renormalized sense. The general formulae for the abstract weak potential with a compact support may have an independent significance for the non-singular cases.

The related problems for the case of presence of the bound states, including the two-dimensional and higher-dimensional spatial delta-model, still await a resolution and proper interpretation. In particular, it represents an emphatic importance in the case of SAE for the conical space with linear angular deficit; it corresponds to the cosmic string. The unperturbative exact solutions for the vacuum polarization effects represent an apparent goal of the subsequent issues.

Author Contributions: Both authors contributed equally. All authors have read and agreed to the published version of the manuscript.

Funding: This research received no external funding.

Data Availability Statement: MDPI Research Data Policies at https:/ / www.mdpi.com/ethics, accessed on 1 May 2021.

Acknowledgments: Authors are grateful to D. Sokolov and D. Fermi for stimulating discussions. The work of P. Spirin was supported by the Scientific and Educational School of Fundamental and Applied Space Research of the Moscow State University.

Conflicts of Interest: The authors declare no conflict of interest.

$\begin{array}{ll}\text { Abbreviations } \\ \text { The following abbreviations are used } \\ \text { FT } & \text { Field Theory } \\ \text { QFT } & \text { Quantum Field Theory } \\ \text { QM } & \text { Quantum Mechanics } \\ \text { PT } & \text { Perturbation Theory } \\ \text { SAE } & \text { Self-Adjoint Extension } \\ \text { EMT } & \text { Energy-Momentum Tensor } \\ \text { VEV } & \text { Vacuum Expectation Value }\end{array}$

\section{References}

1. Kronig, R.L.; Penney W.R. Quantum Mechanics of Electrons in Crystal Lattices. Proc. Roy. Soc. A 1931, $130,499$.

2. Demkov, Y.N.; Ostrovskii, V.N. The Usage of Zero-Range Potentials in Atomic Physics; Nauka: Moscow, Russia, 1975. (In Russian)

3. Mamaev, S.G.; Trunov, N.N. Vacuum Expectation Values of the Energy Momentum Tensor of Quantized Fields on Manifolds with Different Topologies and Geometries III. Sov. Phys. J. 1980, 23, 551. [CrossRef]

4. Mamaev, S.G.; Trunov, N.N. Quantum Effects in the External Fields Determined by the Potentials with Point-like Support. Yad. Fiz. 1982, 35, 1049. (In Russian)

5. Bordag, M.; Henning, D.; Robaschik, D. Vacuum energy in quantum field theory with external potentials concentrated on planes. J. Phys. A 1992, 25, 4483. [CrossRef]

6. Bordag, M.; Mohideen, U.; Mostepanenko, V.M. New developments in the Casimir effect. Phys. Rep. 2001, 353, 1205. [CrossRef]

7. Bordag, M.; Klimchitskaya, G. L.; Mohideen, U.; Mostepanenko, V. M. Advances in the Casimir Effect; Oxford University Press: Oxford, UK, 2009.

8. Muños-Castañeda, J. M.; Moteos Guilarte, J.; Moreno Mosquera, A. Quantum vacuum energies and Casimir forces between partially transparent $\delta$-function plates. Phys. Rev. D 2013, 87, 105020. [CrossRef]

9. Grats, Y.V. Casimir Energy in Contact-Interaction Models. Phys. Atom. Nucl. 2018, 81, 253-256. [CrossRef]

10. Fermi, D.; Pizzocchero, L. Local Casimir Effect for a Scalar Field in Presence of a Point Impurity. Symmetry 2018, 10, 38. [CrossRef] 
11. Fermi, D.; Pizzocchero, L. Local Zeta Regularization and the Scalar Casimir Effect. A General Approach Based on Integral Kernels; World Scientific Publishing Co.: Singapore, 2017.

12. Fermi, D. Vacuum Polarization with Zero-Range Potentials on a Hyperplane. Universe 2021, 7, 92. [CrossRef]

13. Grats, Y.V. Vacuum Polarization in a Zero-Range Potential Field. Phys. Atom. Nucl. 2019, 82, 153-157. [CrossRef]

14. Sokolov, D.D.; Starobinsky, A.A. The structure of the curvature tensor at conical singularities. Sov. Phys. Dokl. 1977, $22,312$.

15. Grats, Y.V.; Spirin, P. Vacuum polarization and classical self-action near higher-dimensional defects. Eur. Phys. J. C 2017, 77, 101. [CrossRef]

16. Grats, Y.V.; Spirin, P. Vacuum polarization in the background of conical singularity. Int. J. Mod. Phys. A 2020, 35, 2040030. [CrossRef]

17. Jackiw, R. Delta function potentials in two-dimensional and three-dimensional quantum mechanics. In M. A. Beg Memorial Volume; Ali, A., Hoodbhoy, P., Eds.; World Scientific: Singapore, 1991; 15p.

18. Jackiw, R.W. Diverse Topics in Theoretical and Mathematical Physics; World Scientific: Singapore, 1995.

19. Allen, B.; Ottewill, A.C. Effects of curvature couplings for quantum fields on cosmic string space-times. Phys. Rev. D 1990, 42, 2669. [CrossRef] [PubMed]

20. Kay, B.; Studer, U.M. Boundary conditions for quantum mechanics on cones and fields around cosmic strings. Comm. Math. Phys. 1991, 139, 103 [CrossRef]

21. Allen, B.; Kay, B.S.; Ottewill, A.C. Long range effects of cosmic string structure. Phys. Rev. D 1996, 53, 6829. [CrossRef]

22. Khusnutdinov, N.R.; Khabibullin, A.R. Zero point energy of a massless scalar field in the cosmic string space-time. Gen. Rel. Grav. 2004, 36, 1613. [CrossRef]

23. Berezin, F.A.; Faddeev, L.D. Remark on the Schrödinger equation with singular potential. Soviet. Math. Dokl. $1961,2,372$.

24. Zel'dovich, Y.B. Scattering by a singular potential in perturbation theory and in the momentum representation. JETP 1960, 11, 594.

25. Solodukhin, S.N. Exact solution for a quantum field with delta-like interaction. Nucl. Phys. B 1999, 541, 461. [CrossRef]

26. Spreafico, M.; Zerbini, S. Finite temperature quantum field theory on noncompact domains and application to delta interactions. Rep. Math. Phys. 2009, 63, 163. [CrossRef]

27. Scardicchio, A. Casimir Dynamics: Interactions of Surfaces with Codimension $>1$ Due to Quantum Fluctuations. Phys. Rev. D 2005, 72, 065004. [CrossRef]

28. Albeverio, S.; Cognola, G.; Spreafico, M.; Zerbini, S. Singular perturbations with boundary conditions and the Casimir effect in the half space. J. Math. Phys. 2010, 51, 063502. [CrossRef]

29. Fermi, D. The Casimir energy anomaly for a point interaction. Mod. Phys. Lett. A 2020, 35, 2040008. [CrossRef]

30. Reed, M.; Simon, B. Methods of Modern Mathematical Physics. II. Fourier Analysis. Self-Adjointness; Academic-Press: New York, NY, USA; London, UK, 1975.

31. Albeverio, S.; Gesztesy, R.; Hoegh-Krohn, R.; Holden, H. Solvable Models in Quantum Mechanics; World Scientific: Singapore, 1995.

32. Gitman, D.M; Tyutin, I.V.; Voronov, B.L. Self-Adjoint Extensions in Quantum Mechanics; Springer: New York, NY, USA, 2012.

33. Birrell, N.D.; Davies, P.C.W. Quantum Fields in Curved Space; Cambridge University Press: Cambridge, UK, 1982.

34. Itzykson, C.; Zuber, J.B. Quantum Field Theory; Mcgraw-Hill: New York, NY, USA, 1980.

35. Gel'fand I.M.; Shilov, G.E. Generalized Functions: Properties and Operations; Academic Press: Waltham, MA, USA, 1964.

36. Christensen, S.M. Vacuum expectation value of the stress tensor in an arbitrary curved background: The covariant point-separation method. Phys. Rev. D 1976, 14, 2490. [CrossRef]

37. Peskin, M.E.; Schroeder, D.V. An Introduction to Quantum Field Theory; Westview Press: Pod, FL, USA, 1995.

38. Gradshteyn, I.S.; Ryzhik, I.M. Table of Integrals, Series, and Products; Academic Press: New York, NY, USA, 2007.

39. Abramowitz, M.; Stegun, I. Handbook of Mathematical Functions with Formulas, Graphs, and Mathematical Tables; U.S. Government Printing Office: Washington, DC, USA, 1964.

40. Park, D.K. Green's-function approach to two- and three-dimensional delta-function potentials and application to the spin-1/2 Aharonov-Bohm problem. J. Math. Phys. 1995, 36, 5453. [CrossRef] 